TRANSACTIONS OF THE

AMERICAN MATHEMATICAL SOCIETY

Volume 353, Number 5 , Pages 2019-2058

S 0002-9947(01)02711-8

Article electronically published on January 10, 2001

\title{
UNCONDITIONAL STRUCTURES OF WEAKLY NULL SEQUENCES
}

\author{
S. A. ARGYROS AND I. GASPARIS
}

\begin{abstract}
The following dichotomy is established for a normalized weakly null sequence in a Banach space: Either every subsequence admits a convex block subsequence equivalent to the unit vector basis of $c_{0}$, or there exists a subsequence which is boundedly convexly complete.
\end{abstract}

\section{INTRODUCTION}

The semi-normalized weakly null sequences (i.e., sequences $\left(x_{j}\right)$ in a Banach space converging weakly to zero and such that $\inf _{j}\left\|x_{j}\right\|>0$ ) being a fundamental concept in the theory of Banach spaces have been studied extensively and several results about their structure, and the structure of the spaces spanned by them have been proved. We mention Bessaga and Pelczynski's theorem [B-P], that any such sequence has a subsequence which is Schauder basic, and Maurey and Rosenthal's examples $[\mathrm{M}-\mathrm{R}]$ of weakly null sequences without unconditional basic subsequences. Both results are fundamental with enormous impact in the development of the theory.

After the appearance of Maurey and Rosenthal's examples, a number of results establishing certain forms of "restricted" unconditionality for weakly null sequences were obtained. We recall Elton's near unconditionality [E], O1, the Schreier unconditionality, stated in [M-R] and also proved later by Odell [O2], and the ArgyrosMercourakis - Tsarpalias convex unconditionality [A-M-T]. Also, the Schreier families $\left\{S_{\xi}\right\}_{\xi<\omega_{1}}[\mathrm{~A}-\mathrm{A}]$, and the repeated averages hierarchy [A-M-T], determined the structure of those convex combinations of a weakly null sequence that tend to zero in norm.

The second and the third sections of the present paper are devoted to a unified approach of these results. Some of them are stated in a more general setting and the proofs, given here, are simpler than the existing ones. The fourth section contains a new dichotomy for weakly null sequences. We shall next explain our results related to this dichotomy and then present the results of the first two sections. We are interested in the isomorphic structure of subsequences of a given sequence $\left(x_{j}\right)$. Therefore, in the sequel, by a weakly null sequence $\left(x_{j}\right)$ we shall mean a normalized

Received by the editors November 20, 1998 and, in revised form, February 14, 2000. 2000 Mathematics Subject Classification. Primary 46B03; Secondary 06A07, 03E10. Key words and phrases. Ramsey theory, weakly null sequence, convex block basis. 
sequence which is additionally bimonotone. That is, $\left\|x_{j}\right\|=1$ and

$$
\sup _{n} \max \left\{\left\|\sum_{i=1}^{n} a_{i} x_{i}\right\|,\left\|\sum_{i=n+1}^{\infty} a_{i} x_{i}\right\|\right\} \leq\left\|\sum_{i=1}^{\infty} a_{i} x_{i}\right\|,
$$

for all choices of scalars $\left(a_{i}\right)$. We shall use standard Banach space facts and terminology. Throughout this paper, $X$ will denote a real infinite dimensional Banach space and $B_{X}$ its closed unit ball. $X^{*}$ stands for the Banach space of real-valued linear functionals on $X$ which are continuous with respect to the norm topology. $c_{0}$ denotes the Banach space of real sequences tending to zero, under the supremum norm. $\ell_{1}$ is the Banach space of absolutely summable real sequences, under the norm given by the sum of the absolute values of the coordinates. We mention here that in the sequel, we shall often identify the elements of $\ell_{1}$ with signed measures on $\mathbb{N}$. A semi-normalized basic sequence $\left(y_{j}\right)$ in $X$ is called $C$ equivalent to the unit vector basis of $c_{0}$, if there exists a positive constant $C$ such that $\left\|\sum_{j=1}^{n} a_{j} y_{j}\right\| \leq C \max _{j \leq n}\left|a_{j}\right|$, for every $n \in \mathbb{N}$, and all choices of scalars $\left(a_{j}\right)_{j=1}^{n}$.

Given $\left(x_{n}\right)$ a sequence in a Banach space, a sequence $\left(y_{n}\right)$ is called a block subsequence of $\left(x_{n}\right)$, if there exist consecutive sets $F_{i} \subset \mathbb{N}$ (that is, $\max F_{i}<$ $\left.\min F_{i+1}, i \in \mathbb{N}\right)$, and a sequence $\left(a_{i}\right)$ of scalars such that for every $i \in \mathbb{N}, y_{i}=$ $\sum_{n \in F_{i}} a_{n} x_{n}$. We then denote by $\operatorname{supp} y_{i}$, the support of $y_{i}$, that is the set $\left\{n \in F_{i}\right.$ : $\left.\left|a_{n}\right|>0\right\}$. We shall also adopt the notation $y_{1}<y_{2}<\cdots$ to indicate that $\left(y_{n}\right)$ is a block subsequence of $\left(x_{n}\right) ;\left(y_{n}\right)$ is called an absolutely convex (resp. sub-convex) block subsequence if $\left(a_{i}\right)$ satisfies $\sum_{n \in F_{i}}\left|a_{n}\right|=1$ (resp. $\sum_{n \in F_{i}}\left|a_{n}\right| \leq 1$ ). If in addition the $a_{i}$ 's are non-negative, then $\left(y_{n}\right)$ is a convex (resp. sub-convex) block subsequence of $\left(x_{n}\right)$.

For an infinite subset $M$ of $\mathbb{N}$, we let $[M]$ (resp. $[M]^{<\infty}$ ) denote the set of its infinite (resp. finite) subsets. $[M]^{k}, k \in \mathbb{N}$, is the subset of $[M]^{<\infty}$ whose members have $k$ elements. Finally, $\omega_{1}$ denotes the first uncountable ordinal. We start with some definitions and notations.

Definition 1.1. 1. A sequence $s=\left(x_{j}\right)$ in a Banach space $X$ is said to be series bounded if

$$
\sup _{n}\left\|\sum_{j=1}^{n} x_{j}\right\| \stackrel{\text { def }}{=} b(s)<\infty .
$$

2. A semi-normalized Schauder basic sequence $s=\left(x_{j}\right)$ is said to be semiboundedly complete, (sb.c.), if for every sequence of scalars $\left(a_{j}\right)$ such that $\left(a_{j} x_{j}\right)$ is series bounded, we have that $\lim _{j} a_{j}=0$.

The basic definition related to our result is the following.

Definition 1.2. A semi-normalized Schauder basic sequence $s=\left(x_{j}\right)$ is said to be boundedly convexly complete, (b.c.c.), provided the following property holds for every sequence of scalars $\left(a_{j}\right)$ such that $\left(a_{j} x_{j}\right)$ is series bounded: Given $\left(F_{j}\right)$, a sequence of consecutive finite subsets of $\mathbb{N}$ such that $\sup _{n} \sum_{j \in F_{n}}\left|a_{j}\right|<\infty$, then $\lim _{n}\left\|\sum_{j \in F_{n}} a_{j} x_{j}\right\|=0$.

It follows easily that every b.c.c. sequence $\left(x_{j}\right)$ is also sb.c. In fact, every seminormalized absolutely convex block subsequence of $\left(x_{j}\right)$ is semi-boundedly complete. We mention here that as a consequence of our main result, Theorem 1.4 
one obtains that every weakly null sequence having the property that every seminormalized absolutely convex block subsequence is semi-boundedly complete, admits a b.c.c. subsequence.

Our next proposition gives a simple characterization of b.c.c. sequences.

Proposition 1.3. Let $s=\left(x_{j}\right)$ be a Schauder basic sequence and let $\mathbf{a}=\left(a_{j}\right)$ be a sequence of scalars. For every $n \in \mathbb{N}$, we define

$$
\tau_{n}(\mathbf{a}, s)=\sup \left\{\left\|\sum_{j \in F} a_{j} x_{j}\right\|: n \leq \min F, \sum_{j \in F}\left|a_{j}\right| \leq 1\right\},
$$

and $\tau(\mathbf{a}, s)=\lim _{n} \tau_{n}(\mathbf{a}, s) . s=\left(x_{j}\right)$ is b.c.c. if and only if, for every sequence of scalars $\mathbf{a}=\left(a_{j}\right)$ such that $\left(a_{j} x_{j}\right)$ is series bounded we have that $\tau(\mathbf{a}, s)=0$.

We omit the easy proof and observe that for a sequence $s=\left(x_{j}\right)$ we have that $\tau(\mathbf{a}, s)>0$ if and only if there exists a sequence $\left(F_{n}\right)$ of consecutive finite subsets of $\mathbb{N}$ such that $\sum_{j \in F_{n}}\left|a_{j}\right| \leq 1$, for all $n \in \mathbb{N}$, and $\inf _{n}\left\|\sum_{j \in F_{n}} a_{j} x_{j}\right\|>0$.

Assuming $s$ is weakly null and that $\mathbf{a}=\left(a_{j}\right)$ satisfies the stronger condition that the series $\sum_{j=1}^{\infty} a_{j} x_{j}$ converges in norm, it does not seem clear that $\tau(\mathbf{a}, s)=0$. However, if $\left(x_{j}\right)$ is convexly unconditional [A-M-T, it is guaranteed that for any such a we have that $\tau(\mathbf{a}, s)=0$. The main result of the fourth section is the following.

Theorem 1.4. For every weakly null sequence $\left(x_{j}\right)$ one of the following two alternatives holds exclusively:

1. There exists a boundedly convexly complete subsequence.

2. Every subsequence admits a convex block subsequence equivalent to the unit vector basis of $c_{0}$.

We recall that as a consequence of Elton's nearly unconditional theorem, one obtains a similar dichotomy for weakly null sequences where the two alternatives go as follows:

1. There exists a semi-boundedly complete subsequence.

2. Every subsequence admits a further subsequence equivalent to the unit vector basis of $c_{0}$.

Thus, our result may be considered as the continuation of Elton's theorem in the direction of a deeper search in the span of the sequence $\left(x_{j}\right)$, for the existence of a block subsequence equivalent to the unit vector basis of $c_{0}$. Also, the alternative to the latter case is the existence of a restricted form of unconditionality which is, in an asymptotic sense, the near unconditionality for convex block subsequences.

Let us observe that if we assume that the sequence $\left(x_{j}\right)$ is unconditional basic, then Theorem 1.4 follows easily by well known arguments. However, even in this case, our proof derives more information about the structure of the sequence. This is a consequence of our next result.

Theorem 1.5. Let $s=\left(x_{n}\right)$ be a weakly null sequence having no subsequence which is b.c.c. There exist $N \in[\mathbb{N}], \xi<\omega_{1}$ and a constant $C>0$ such that the subsequence $\left(x_{n}\right)_{n \in N}$ is an $\ell_{1}^{\xi}$ spreading model, and for every $Q \in[N],\left(\xi_{n}^{Q} \cdot s\right)_{n \in \mathbb{N}}$ is $C$-equivalent to the unit vector basis of $c_{0}$.

In the above, $\left(\xi_{n}^{Q} \cdot s\right)_{n \in \mathbb{N}}$ is the sequence of repeated averages of order $\xi$ of the sequence $\left(x_{n}\right)_{n \in Q}$. This concept will be explained in the next section. 
Therefore, every weakly null sequence with no b.c.c. subsequence, has a subsequence which behaves similarly to the basis of Schreier's space $X_{\xi}$, for some countable ordinal $\xi$. Recall that $X_{\xi}$ is defined as the completion of $\left(c_{00},\|\cdot\|_{\xi}\right)$ where

$$
\|x\|_{\xi}=\sup _{F \in S_{\xi}} \sum_{n \in F}|x(n)|
$$

for $x=(x(n)) \in c_{00}$, the space of ultimately vanishing sequences. We do not know if the subsequence $\left(x_{n}\right)_{n \in M}$ resulting from Theorem 1.5 is actually equivalent to a subsequence of the unit vector basis of $X_{\xi}$ for the corresponding ordinal $\xi$.

The statement of Theorem 1.4 reminds us of Rosenthal's remarkable dichotomy $[\mathrm{R}]$, for non-trivial weak-Cauchy sequences. (A weak Cauchy sequence is called non-trivial if it is non-weakly convergent.) We recall the statement of this theorem.

Theorem 1.6. Every non-trivial weak Cauchy sequence in a (real or complex) Banach space has either a strongly summing subsequence or a convex block basis equivalent to the summing basis.

Following $\left[\mathbf{R}\right.$, a weak Cauchy basic sequence $\left(x_{n}\right)$ is said to be strongly summing (s.s.) provided that the scalar series $\sum_{n} a_{n}$ converges whenever $\sup _{n}\left\|\sum_{i=1}^{n} a_{i} x_{i}\right\|$ is finite. We also recall that the basic sequence $\left(x_{n}\right)$ is equivalent to the summing basis provided that for every choice of scalars $\left(a_{n}\right)$, the series $\sum_{n=1}^{\infty} a_{n} x_{n}$ converges if and only if the series $\sum_{n} a_{n}$ converges.

There are similarities but also differences between Rosenthal's dichotomy and ours. Their relation is discussed in detail in the last section of this paper where we also give a slight improvement of Rosenthal's result, Corollary 5.3 and establish the corresponding statement to Elton's dichotomy for non-trivial weak Cauchy sequences, Corollary 5.5.

The proof of Theorem 1.5 which immediately implies Theorem 1.4, is given in section 4 following Lemma 4.7. Our argument is of combinatorial nature depending heavily on results obtained in sections 2 and 3. Roughly speaking, the nature of this theorem enforces a delicate combination of the near unconditionality with the convex unconditionality and the results related to summability methods. Next we shall give a rough sketch of the proof of Theorem 1.5, indicating how the results obtained in Sections 2 and 3 are used in the argument.

Sketch of the proof of Theorem 1.5. Start with a normalized weakly null sequence $s=\left(x_{n}\right)$ and suppose that no subsequence of $s$ is b.c.c. We can assume that $s$ has no subsequence equivalent to the unit vector basis of $c_{0}$, or else the assertion of Theorem [1.5 trivially holds. Furthermore, Elton's dichotomy, Corollary 3.3 allows us to assume that the sequence $\left(x_{n}^{*}\right)$ of functionals biorthogonal to $\left(x_{n}\right)$ is weakly null in $\left[\left(x_{n}\right)\right]^{*}$. This fact is crucial for establishing the key Lemma 4.6. We shall effectively construct a convex block subsequence of $\left(x_{n}\right)$ equivalent to the unit vector basis of $c_{0}$.

The first step is an easy stabilization argument given in Lemma 4.1. We obtain $M_{0} \in[\mathbb{N}]$ and $\delta_{0}>0$ with the property that for every $L \in\left[M_{0}\right]$ there exist a scalar sequence $\left(a_{m}\right)_{m \in L}$ such that $\left(a_{m} x_{m}\right)_{m \in L}$ is series bounded by 1 , and consecutive subsets $\left(F_{i}\right)$ of $L$ so that for every $i \in \mathbb{N}\left(a_{m}\right)_{m \in F_{i}}$ is sub-convex with $\left\|\sum_{m \in F_{i}} a_{m} x_{m}\right\|>\delta_{0}$.

The main difficulty now is that changing from a subset $L$ of $M_{0}$ to another subset $N$, the sub-convex block subsequence resulting from Lemma4.1 applied on $L$ 
changes arbitrarily to a sub-convex block subsequence supported by $N$. Therefore, there is no obvious way to directly apply the infinite Ramsey theorem (Theorem 2.8). In order to overcome this difficulty, we make strong use of results on the ordinal complexity of weakly null sequences $[\mathrm{A}-\mathrm{A}], \mathrm{A}-\mathrm{M}-\mathrm{T}]$. We apply Theorem 2.13, Corollary 3.6 and Theorem 1.11 in Lemma 4.5, to obtain $M \in[\mathbb{N}], \delta>0$ and $1 \leq \xi<\omega_{1}$ satisfying the following properties:

1. $\left(x_{m}\right)_{m \in M}$ is an $\ell_{1}^{\xi}$ spreading model (Definition 2.1), yet no subsequence of $\left(x_{m}\right)_{m \in M}$ is an $\ell_{1}^{\xi+1}$ spreading model.

2. For every $N \in[M]$ we can find $P \in[N]$ so that for every finite sequence $\left(F_{i}\right)_{i=1}^{n}$ of consecutive maximal $S_{\xi}$ subsets of $P$ there exists a scalar sequence $\left(a_{m}\right)_{m \in N}$ which is $\xi$-faithful for $\left(\delta,\left(F_{i}\right)_{i=1}^{n}\right)$.

The latter means that

(i) $\left(a_{m} x_{m}\right)_{m \in N}$ is series bounded by 1 and $\left|a_{m}\right| \leq \frac{\delta}{2}$, for every $m \in N$.

(ii) $\left(a_{m}\right)_{m \in F_{i}}$ is sub-convex and $\left\|\sum_{m \in F_{i}} a_{m} x_{m}\right\|>\delta$, for all $i \leq n$.

It is a crucial fact for the entire proof that the first ordinal $\alpha$ such that $\left(x_{n}\right)$ admits no subsequence which is an $\ell_{1}^{\alpha}$ spreading model, is a successor ordinal $\alpha=$ $\xi+1$. This is the content of condition 1 above. On the other hand, condition 2 indicates that the semi-normalized convex block subsequence of $\left(x_{n}\right)$ we are seeking, is related to the ordinal $\xi$. Indeed, our goal is to show that for some $L \in[M]$ the sequence $\left(\xi_{i}^{L} \cdot s\right)$ of repeated averages of order $\xi$ of $s$, is actually equivalent to the unit vector basis of $c_{0}$. The repeated averages hierarchy $\left\{\left(\xi_{n}^{L}\right): L \in[\mathbb{N}]\right\}$, enjoys very strong stability properties (P3 and P4 after the definition of the hierarchy) and for this reason it can be handled through Ramsey's theorem and generalizations of Elton's result (Theorem 1.9).

We next apply the combinatorial result of $\mathrm{AN}-\mathrm{O}$ (Lemma 2.4 in our paper) to deduce that the sequence $\left(\xi_{i}^{L} \cdot s\right)$ is uniformly semi-normalized for all $L \in[M]$. The heart of the proof lies in establishing the following:

Given $\lambda>0, \epsilon>0$ and $N \in[M]$, there exist $P, Q$ and $L$ in $[N], L \subset Q \subset P$, so that the following property is fulfilled:

If there exist $y^{*} \in B_{X^{*}}$ and $n \in \mathbb{N}$ such that $\sum_{i=1}^{n} y^{*}\left(\xi_{i}^{L} \cdot s\right)>\lambda$, then there exist $x^{*} \in B_{X^{*}}$, a scalar sequence $\left(a_{m}\right)_{m \in P}$ and consecutive maximal $S_{\xi}$ subsets $\left(F_{i}\right)_{i=1}^{n}$ of $Q$ satisfying:

1. $\sum_{i=1}^{n} x^{*}\left(\xi_{i}^{L} \cdot s\right)>\lambda$, while $\sum_{m \in P \backslash \bigcup_{i=1}^{n} \operatorname{supp} \xi_{i}^{L}}\left|x^{*}\left(x_{m}\right)\right|<\epsilon$ (Lemma 3.2).

2. $\left(a_{m}\right)_{m \in P}$ is $\xi$-faithful for $\left(\delta,\left(F_{i}\right)_{i=1}^{n}\right)$ (Lemma 4.5).

3. $\sum_{m \in P \backslash \bigcup_{i=1}^{n} F_{i}}\left|x^{*}\left(x_{m}\right)\right|<2 \epsilon$ (Lemma 4.6 combined with 1 above).

4. $F_{i} \backslash\left\{\min F_{i}\right\} \subset\left\{m \in \operatorname{supp} \xi_{i}^{L}: x^{*}\left(x_{m}\right) \geq \frac{1}{2} x^{*}\left(\xi_{i}^{L} \cdot s\right)\right\}, i \leq n$ (Lemma 4.7 combined with Proposition 2.15).

Conditions 1-4 yield that for $\lambda>\frac{5}{\delta}$ and $N \in[M]$, there exists $L \in[N]$ so that $\left\|\sum_{i=1}^{n} \xi_{i}^{L} \cdot s\right\| \leq \lambda$, for all $n \in \mathbb{N}$. We deduce from the infinite Ramsey theorem, that for some $L \in[M],\left(\xi_{i}^{L} \cdot s\right)$ is equivalent to the unit vector basis of $c_{0}$.

We next proceed with the results of the first two sections of this paper. In section 2 we present a criterion for embedding the family $S_{\xi}^{n}(M)$, Definition 2.3 where $M \in[\mathbb{N}], \xi<\omega_{1}$ and $n \in \mathbb{N}$, into a hereditary family $\mathfrak{F}$ of finite subsets of $\mathbb{N}$. This criterion, Theorem 2.13 is related to the notion of $(\xi, M, \delta, n)$ large families, Definition [2.16, introduced in [A-M-T] and [A-F], and for the case $n=1$ it roughly says that given a hereditary family $\mathfrak{F}$ of finite subsets of $\mathbb{N}$, there exists $M \in[\mathbb{N}]$ with $S_{\xi}(M)$ contained in $\mathfrak{F}$ provided the following property holds for some subset 
$\mathcal{A}$ of the probability measures on $\mathbb{N}: \sup _{F \in \mathfrak{F}} \mu(F)>\delta>0$, for all $\mu \in \mathcal{A}$, and for every $L \in[\mathbb{N}]$, every $\epsilon>0$ and $\zeta<\xi$, there exists $\mu \in \mathcal{A}$ supported by $L$ and such that $\sup _{F \in S_{\zeta}} \mu(F)<\epsilon$. We apply Theorem 2.13 in section 3 in order to obtain a simpler proof (bypassing the use of the strong Cantor-Bendixson index) of the following dichotomy established in [A-M-T].

Theorem 1.7. For a weakly null sequence $s=\left(x_{n}\right)$ in a Banach space and $1 \leq$ $\xi<\omega_{1}$, one of the following holds exclusively:

1. $\left(x_{n}\right)$ is $\xi$-convergent.

2. There exists $M \in[\mathbb{N}], M=\left(m_{i}\right)_{i \in \mathbb{N}}$, such that $\left(x_{m_{i}}\right)$ is an $l_{1}^{\xi}$ spreading model.

$\xi$-convergence is introduced in Definition [2.2. The concept of an $l_{1}^{\xi}$ spreading model is given in Definition 2.1.

It is perhaps worth noting that the summability methods introduced in A-M-T] form the natural ordinal analogs of the Cesaro summability. These methods have been already employed in modern Banach space theory, $[\mathrm{A}-\mathrm{F}], \mathrm{O}-\mathrm{S}]$ and it is possible that they can be applied to other branches of mathematics as well. The proof of Theorem 1.7 given here is accessible to non-specialists.

Theorem 1.7 yields

Corollary 1.8. Let $\left(x_{n}\right)$ be a weakly null sequence which is an $l_{1}^{\xi}$ spreading model for some ordinal $\xi<\omega_{1}$, yet no subsequence of $\left(x_{n}\right)$ is an $l_{1}^{\xi+1}$ spreading model. Then there exists a semi-normalized convex block subsequence of $\left(x_{n}\right)$ which is Cesaro summable.

Let us remark here that the hypothesis of Corollary[1.8 is satisfied for any weakly null sequence in a Banach space whose Szlenk index [Sz], is equal to $\omega^{n}, n \in \mathbb{N}$. Thus we generalize the result of Alspach and Odell, $\mathrm{A}-\mathrm{O}$, who established Corollary 1.8 for weakly null sequences in $C\left(\omega^{\omega^{n}}\right), n \in \mathbb{N}$.

In the third section we give a simpler proof and a generalization of Elton's nearly unconditional theorem, [E], O1]. More precisely we show

Theorem 1.9. Let $s=\left(x_{n}\right)$ be a weakly null sequence in a Banach space and $\xi<\omega_{1}$. There exists $M \in[\mathbb{N}]$ such that for every $\delta \in(0,1]$ there exists a constant $C(\delta)>0$ so that the following property is satisfied: If $L \in[M], n \in \mathbb{N}$ and $\left(a_{i}\right)_{i=1}^{n}$ are scalars in $[-1,1]$, then

$$
\left\|\sum_{i \in F} a_{i}\left(\xi_{i}^{L} \cdot s\right)\right\| \leq C(\delta) \max \left\{\delta,\left\|\sum_{i=1}^{n} a_{i}\left(\xi_{i}^{L} \cdot s\right)\right\|\right\},
$$

for all $F \subset\left\{i \leq n:\left|a_{i}\right| \geq \delta\right\}$.

The proof of Theorem 1.9 is based on the combinatorial Lemma 3.2 The dual version of this lemma, Lemma 3.4 readily implies that every normalized weakly null sequence admits a convexly unconditional subsequence. This is one of the main results in $[\mathrm{A}-\mathrm{M}-\mathrm{T}]$. It can be equivalently formulated as follows:

Theorem 1.10. Let $\left(x_{n}\right)$ be a weakly null sequence in a Banach space. There exists $M \in[\mathbb{N}], M=\left(m_{i}\right)$, such that for all $\delta>0$, there exists a constant $C(\delta)>0$ so that the following property is satisfied: If $F \in[\mathbb{N}]^{<\infty}$ and $\left(\lambda_{i}\right)_{i \in F}$ are scalars with $\left\|\sum_{i \in F} \lambda_{i} x_{m_{i}}\right\|>\delta$ and $\sum_{i \in F}\left|\lambda_{i}\right| \leq 1$, then $\left\|\sum_{i=1}^{\infty} a_{i} x_{m_{i}}\right\|>C(\delta)$, for all choices of scalars $\left(a_{i}\right)_{i=1}^{\infty} \subset c_{00}$, with $\max _{i}\left|a_{i}\right| \leq 1$ and such that $\left|a_{i}\right|=\left|\lambda_{i}\right|$, for all $i \in F$. 
Another application of Lemma 3.4 is on the $S_{\xi}$ unconditionality of $l_{1}^{\xi}$ spreading models. Recall that the sequence $\left(x_{n}\right)$ is said to be $S_{\xi}$ unconditional, if there exists a constant $C>0$ such that

$$
\left\|\sum_{i \in F} a_{i} x_{i}\right\| \leq C\left\|\sum_{i=1}^{\infty} a_{i} x_{i}\right\|
$$

for every $F \in S_{\xi}$ and all choices of scalars $\left(a_{i}\right)_{i=1}^{\infty} \subset c_{00}$.

Theorem 1.11. Let $\left(x_{n}\right)$ be a weakly null sequence and $\xi<\omega_{1}$. Assume that $\left(x_{n}\right)$ is an $l_{1}^{\xi}$ spreading model. There exists $M \in[\mathbb{N}], M=\left(m_{i}\right)$, such that $\left(x_{m_{i}}\right)$ is $S_{\xi}$ unconditional.

We also obtain Corollary 3.7, the result on Schreier unconditionality [M-R, O2], that every normalized weakly null sequence admits, for every $\epsilon>0$, a subsequence which is $2+\epsilon S_{1}$ unconditional.

Our final results, Theorem 1.12 and Corollary 1.13 , concern the duality between $c_{0}{ }^{\xi}$ (Definition [3.8) and $l_{1}^{\xi}$ spreading models, and the concept of the hereditary $\xi$ Dunford-Pettis property (Definition 3.9).

Notation. If $M \in[\mathbb{N}]$ and $\left(x_{n}\right)$ is a sequence in $X$, then we let $X_{M}$ denote the closed linear span of the subsequence $\left(x_{n}\right)_{n \in M}$.

The duality between $c_{0}^{\xi}$ and $\ell_{1}^{\xi}$ spreading models is described next.

Theorem 1.12. Let $\left(x_{n}\right)$ and $\left(x_{n}^{*}\right)$ be normalized weakly null sequences in $X$ and $X^{*}$ respectively. Assume that for some $\epsilon>0$ we have that $\inf _{n}\left|x_{n}^{*}\left(x_{n}\right)\right| \geq \epsilon$. Let $1 \leq \xi<\omega_{1}$. The following are equivalent:

1. For every $M \in[\mathbb{N}]$, there exists $L \in[M]$ such that $\left(x_{n}^{*} \mid X_{M}\right)_{n \in L}$ is an $\ell_{1}^{\xi}$ spreading model in $X_{M}^{*}$.

2. For every $M \in[\mathbb{N}]$, there exists $L \in[M]$ such that $\left(x_{n}\right)_{n \in L}$ is a $c_{0}^{\xi}$ spreading model.

Corollary 1.13. For a Banach space $X$ and $1 \leq \xi<\omega_{1}$, the following are equivalent:

1. Every normalized weakly null sequence in $X$ admits a subsequence which is a $c_{0}^{\xi}$ spreading model.

2. $X$ is hereditarily $\xi-D P$.

Before closing this section we would like to mention that according to an unpublished result of Johnson, O1, if every subsequence of a normalized weakly null sequence $\left(x_{n}\right)$ admits a further subsequence which is series bounded, then there exists a subsequence of $\left(x_{n}\right)$ equivalent to the unit vector basis of $c_{0}$. Theorem 1.4 immediately yields a generalization of Johnson's result as the following corollary shows.

Corollary 1.14. Let $\left(x_{n}\right)$ be a weakly null sequence in a Banach space. Assume that every subsequence of $\left(x_{n}\right)$ admits a semi-normalized convex block subsequence which is series bounded. Then there exists a convex block subsequence of $\left(x_{n}\right)$ equivalent to the unit vector basis of $c_{0}$.

We must point out here that even under the stronger assumption of Corollary 1.14, the proof has to go through the arguments of the general case.

We wish to thank H. Rosenthal for useful discussions regarding this paper. 


\section{LARGE FAMILIES}

In this section we present a criterion, Theorem 2.13, for embedding the family $S_{\xi}^{n}(M)$, where $M \in[\mathbb{N}], \xi<\omega_{1}$ and $n \in \mathbb{N}$, into a hereditary family $\mathfrak{F}$ of finite subsets of $\mathbb{N}$. This criterion will be applied in section 3 in the proof of Theorem 1.7.

We shall now introduce some notation and terminology that will be frequently used in the sequel and state all necessary definitions of the concepts discussed in this paper. We first recall the definition of the generalized Schreier families introduced in $\mathrm{A}-\mathrm{A}$. It is convenient here to associate to each countable ordinal $\xi$, a sequence of successor ordinals $\left(\xi_{n}+1\right)$ such that $\xi_{n}+1=\xi$, for all $n \in \mathbb{N}$, if $\xi$ is a successor ordinal, while $\left(\xi_{n}+1\right)$ strictly increases to $\xi$, if $\xi$ is a limit ordinal. In the sequel we shall refer to $\left(\xi_{n}+1\right)$ as the sequence of ordinals associated to $\xi$.

Notation. If $F_{1}, F_{2}$ are non-empty subsets of $\mathbb{N}$ with $F_{1}$ finite, we denote by $F_{1}<F_{2}$ the relation $\max F_{1}<\min F_{2}$. If $\mu, \nu$ are finitely supported signed measures on $\mathbb{N}$, we also write $\mu<\nu$ if $\operatorname{supp} \mu<\operatorname{supp} \nu$.

The Schreier families. $\left\{S_{\xi}\right\}_{\xi<\omega_{1}}$ are defined by transfinite induction as follows:

$$
S_{0}=\{\{n\}: n \in \mathbb{N}\} \cup\{\emptyset\} .
$$

Suppose that the families $S_{\alpha}$ have been defined for all $\alpha<\xi$.

If $\xi=\zeta+1$, we set

$$
S_{\xi}=\left\{F \in[\mathbb{N}]^{<\infty}: F=\bigcup_{i=1}^{n} F_{i}, F_{i} \in S_{\zeta}, i \leq n, n \leq F_{1}<\cdots<F_{n}\right\} \cup\{\emptyset\} .
$$

If $\xi$ is a limit ordinal, let $\left(\xi_{n}+1\right)$ be the sequence of ordinals associated to $\xi$ and set

$$
S_{\xi}=\left\{F \in[\mathbb{N}]^{<\infty}: n \leq \min F, \text { and } F \in S_{\xi_{n}+1}, \text { for some } n \in \mathbb{N}\right\} .
$$

Definition 2.1. A sequence $\left(x_{n}\right)$ in a Banach space is an $l_{1}^{\xi}$ spreading model, $\xi<\omega_{1}$, if there exists a constant $C>0$ such that

$$
\left\|\sum_{i \in F} a_{i} x_{i}\right\| \geq C \sum_{i \in F}\left|a_{i}\right|
$$

for every $F \in S_{\xi}$ and all choices of scalars $\left(a_{i}\right)_{i \in F}$.

The repeated averages hierarchy. This hierarchy was introduced in $\mathrm{A}-\mathrm{M}-\mathrm{T}$. We let $\left(e_{n}\right)$ denote the unit vector basis of $\ell_{1}$. For every countable ordinal $\xi$ and every $M \in[\mathbb{N}]$, we define a convex block subsequence $\left(\xi_{n}^{M}\right)_{n=1}^{\infty}$ of $\left(e_{n}\right)$ by transfinite induction on $\xi$ in the following manner: If $\xi=0$, then $\xi_{n}^{M}=e_{m_{n}}$, for all $n \in \mathbb{N}$, where $M=\left(m_{n}\right)$. Assume that $\left(\zeta_{n}^{M}\right)_{n=1}^{\infty}$ has been defined for all $\zeta<\xi$ and $M \in[\mathbb{N}]$. Let $\xi=\zeta+1$. Set

$$
\xi_{1}^{M}=\frac{1}{m_{1}} \sum_{i=1}^{m_{1}} \zeta_{i}^{M}
$$

where $m_{1}=\min M$. Suppose that $\xi_{1}^{M}<\cdots<\xi_{n}^{M}$ have been defined. Let

$$
M_{n}=\left\{m \in M: m>\max \operatorname{supp} \xi_{n}^{M}\right\} \text { and } k_{n}=\min M_{n} .
$$


Set

$$
\xi_{n+1}^{M}=\frac{1}{k_{n}} \sum_{i=1}^{k_{n}} \zeta_{i}^{M_{n}}
$$

If $\xi$ is a limit ordinal, let $\left(\xi_{n}+1\right)$ be the sequence of ordinals associated to $\xi$, and let $M \in[\mathbb{N}]$. Define

$$
\xi_{1}^{M}=\left[\xi_{m_{1}}+1\right]_{1}^{M}
$$

where $m_{1}=\min M$. Suppose that $\xi_{1}^{M}<\cdots<\xi_{n}^{M}$ have been defined. Let

$$
M_{n}=\left\{m \in M: m>\max \operatorname{supp} \xi_{n}^{M}\right\} \text { and } k_{n}=\min M_{n} .
$$

Set

$$
\xi_{n+1}^{M}=\left[\xi_{k_{n}}+1\right]_{1}^{M_{n}} .
$$

The inductive definition of $\left(\xi_{n}^{M}\right)_{n=1}^{\infty}, M \in[\mathbb{N}]$ is now complete. The following properties are established in $\mathrm{A}-\mathrm{M}-\mathrm{T}$.

P1: $\left(\xi_{n}^{M}\right)_{n=1}^{\infty}$ is a convex block subsequence of $\left(e_{n}\right)$ and $M=\bigcup_{n=1}^{\infty} \operatorname{supp} \xi_{n}^{M}$ for all $M \in[\mathbb{N}]$ and $\xi<\omega_{1}$.

P2: $\operatorname{supp} \xi_{n}^{M} \in S_{\xi}$, for all $M \in[\mathbb{N}], \xi<\omega_{1}$ and $n \in \mathbb{N}$.

P3: If $M, N \in[\mathbb{N}], \xi<\omega_{1}$, and $\operatorname{supp} \xi_{i}^{M}=\operatorname{supp} \xi_{i}^{N}$, for $i \leq k$, then $\xi_{i}^{M}=\xi_{i}^{N}$ for $i \leq k$.

P4: If $\xi<\omega_{1},\left\{n_{k}: k \in \mathbb{N}\right\} \subset \mathbb{N}$, and $\left\{L_{k}: k \in \mathbb{N}\right\} \subset[\mathbb{N}]$, are such that $\operatorname{supp} \xi_{n_{i}}^{L_{i}}<\operatorname{supp} \xi_{n_{i+1}}^{L_{i+1}}$, for all $i \in \mathbb{N}$, then letting $L=\bigcup_{i=1}^{\infty} \operatorname{supp} \xi_{n_{i}}^{L_{i}}$, we have that $\xi_{i}^{L}=\xi_{n_{i}}^{L_{i}}$, for all $i \in \mathbb{N}$.

Properties $\mathbf{P} \mathbf{3}$ and $\mathbf{P} 4$ are called stability properties of the hierarchy $\left\{\left(\xi_{n}^{M}\right)_{n=1}^{\infty}\right.$ : $M \in[\mathbb{N}]\}$.

Next we recall the definition of $(M, \xi)$ convergent sequences.

Notation. For a sequence $s=\left(x_{n}\right)$ in a Banach space and a vector $\mu=\sum_{n=1}^{\infty} a_{n} e_{n}$ of $\ell_{1}$, we set $\mu \cdot s=\sum_{n=1}^{\infty} a_{n} x_{n}$.

Definition 2.2. A sequence $s=\left(x_{n}\right)$ in a Banach space is called $(M, \xi)$ convergent, $\xi<\omega_{1}, M \in[\mathbb{N}]$, if $\lim _{n}\left\|\xi_{n}^{M} \cdot s\right\|=0$. The sequence $s$ is called $\xi$-convergent, if for every $M \in[\mathbb{N}]$, there exists $N \in[M]$ such that $s$ is $(L, \xi)$ convergent, for all $L \in[N]$.

Remark. A sequence is $(M, \xi+1)$ convergent if and only if it is $(M, \xi)$ summable. The latter concept is defined in [A-M-T]. Therefore, the $\xi+1$-convergence of a sequence is equivalent to its $\xi$-summability introduced in [A-M-T].

We continue this preliminary discussion with the notion of a hereditary family.

Definition 2.3. $\quad$ 1. A family $\mathfrak{F}$ of finite subsets of $\mathbb{N}$ is called hereditary, if for every $G \in \mathfrak{F}$ and $F \subset G$ we have that $F \in \mathfrak{F}$.

2. If $\mathfrak{F}$ is hereditary and $M \in[\mathbb{N}], M=\left(m_{i}\right)$, then we define $\mathfrak{F}[M]=\{F \in \mathfrak{F}$ : $F \subset M\}$, and $\mathfrak{F}(M)=\{M(F): F \in \mathfrak{F}\}$, where $M(F)=\left\{m_{i}: i \in F\right\}$.

3. If $\mathfrak{F}$ is hereditary and $n \in \mathbb{N}$, then we set

$$
\mathfrak{F}^{n}=\left\{\bigcup_{i=1}^{n} F_{i}: F_{1}<\cdots<F_{n} \text { and } F_{i} \in \mathfrak{F}, i \leq n\right\} .
$$


Remark. Evidently, if $\mathfrak{F}$ is hereditary, then so are the families $\mathfrak{F}[M], \mathfrak{F}(M)$ and $\mathfrak{F}^{n}$ for every $n \in \mathbb{N}$ and $M \in[\mathbb{N}]$. It is also easily seen, by induction, that the Schreier families $\left\{S_{\xi}\right\}_{\xi<\omega_{1}}$ are hereditary.

We observe that for all $\xi<\omega_{1}$ and $M \in[\mathbb{N}], S_{\xi}(M) \subset S_{\xi}[M]$. Note that the inverse inclusion does not hold. However, the following result of Androulakis and Odell [AN-O], shows that the two families are in a certain sense comparable.

Lemma 2.4. For every $M \in[\mathbb{N}]$ there exists $N \in[M]$ such that for all $\xi<\omega_{1}$, $\left\{F \backslash\{\min F\}: F \in S_{\xi}[N]\right\} \subset S_{\xi}(M)$.

The next three lemmas describe properties of the maximal members of $S_{\xi}, \xi<$ $\omega_{1}$. Their proofs are easily obtained by transfinite induction and therefore we omit them.

Lemma 2.5. Let $\xi<\omega_{1}$ and $F \in S_{\xi}$. The following are equivalent:

1. If $F \subset G$ and $G \in S_{\xi}$, then $F=G$. Thus, $F$ is a maximal $S_{\xi}$ set.

2. $F \cup\{n\} \notin S_{\xi}$, for all $n \in \mathbb{N}$ with $\max F<n$.

3. $F \cup\{n\} \notin S_{\xi}$, for some $n \in \mathbb{N}$ with $\max F<n$.

Lemma 2.6. Suppose that $F_{1}<\cdots<F_{n}$ belong to $S_{\xi}[M], M \in[\mathbb{N}]$. There exist $k \leq n$ and $G_{1}<\cdots<G_{k}$, maximal members of $S_{\xi}[M]$ with $\min G_{1}=\min F_{1}$ and such that

$$
\bigcup_{i=1}^{k-1} G_{i} \subset \bigcup_{i=1}^{n} F_{i} \subset \bigcup_{i=1}^{k} G_{i}, \quad\left(G_{0}=\emptyset\right) .
$$

Lemma 2.7. Let $M \in[\mathbb{N}], \xi<\omega_{1}$. There exists a (necessarily) unique sequence $\left\{F_{n}^{\xi}(M)\right\}_{n \in \mathbb{N}}$ of consecutive maximal $S_{\xi}$ sets such that

$$
M=\bigcup_{n=1}^{\infty} F_{n}^{\xi}(M)
$$

Remark. 1. It is easily seen that if $N=\bigcup_{n=1}^{\infty} F_{k_{n}}^{\xi}(M)$, where $k_{1}<k_{2}<\cdots$, then $F_{n}^{\xi}(N)=F_{k_{n}}^{\xi}(M)$, for all $n \in \mathbb{N}$.

2. Let $M=\left(m_{i}\right)$ and $N=\left(n_{i}\right)$ be infinite subsets of $\mathbb{N}$. Assume that for some $p \in \mathbb{N}$, we have that $m_{i}=n_{i}$, for all $i \leq p$. If $F_{k}^{\xi}(M)$ is contained in $\left\{m_{i}: i \leq p\right\}$, then, $F_{i}^{\xi}(M)=F_{i}^{\xi}(N)$, for all $i \leq k$.

Notation. We let $\mathcal{M}$ denote the set of those signed measures on $\mathbb{N}$ whose variation does not exceed 1 . Clearly, $\mathcal{M}$ is naturally identified with the ball of $\ell_{1}$. If $\mu \in \mathcal{M}$ and $\xi<\omega_{1}$, we set

$$
\|\mu\|_{\xi}=\sup \left\{|\mu|(F): F \in S_{\xi}\right\} .
$$

We would like to mention here that the proofs of the results of this paper rely essentially on an important principle of infinite combinatorics known as the infinite Ramsey theorem. This theorem was proved in several steps by Nash-Williams NW], Galvin and Prikry G-P and Silver SSi. Proofs of the infinite Ramsey theorem which are not model-theoretic, were given by Ellentuck [Ell, and recently by Tsarpalias $[\mathrm{T}$. We recall the statement of the theorem. $[\mathbb{N}]$ is endowed with the topology of the pointwise convergence.

Theorem 2.8. Let $A$ be an analytic subset of $[\mathbb{N}]$. For every $M \in[\mathbb{N}]$ there exists $L \in[M]$ such that either $[L] \subset A$, or, $[L] \subset[M] \backslash A$. 
In the sequel, any set satisfying the conclusion of Theorem [2.8, will be called (completely) Ramsey.

Our next lemma is crucial for the proof of Theorem 2.13.

Lemma 2.9. Let $\mathfrak{F}$ be a hereditary family of finite subsets of $\mathbb{N}, \xi$ a countable ordinal and $m \in \mathbb{N}$. For every $M \in[\mathbb{N}]$ there exists $N \in[M]$ such that either $\bigcup_{i=1}^{m} F_{i}^{\xi}(L) \in \mathfrak{F}$ for all $L \in[N]$, or, $\bigcup_{i=1}^{m} F_{i}^{\xi}(L) \notin \mathfrak{F}$ for all $L \in[N]$.

Proof. Let

$$
\Delta=\left\{L \in[M]: \bigcup_{i=1}^{m} F_{i}^{\xi}(L) \in \mathfrak{F}\right\}
$$

It follows by the second remark after Lemma 2.7, that $\Delta$ is closed in $[M]$ and therefore Ramsey. Hence, there exists $N \in[M]$ such that either $[N] \subset \Delta$, or, $[N] \cap \Delta=\emptyset$. If the former, then $\bigcup_{i=1}^{m} F_{i}^{\xi}(L) \in \mathfrak{F}$, for all $L \in[N]$. If the latter, then $\bigcup_{i=1}^{m} F_{i}^{\xi}(L) \notin \mathfrak{F}$, for all $L \in[N]$.

Definition 2.10. Suppose that $\mathcal{A} \subset \mathcal{M}, \mathfrak{F}$ is a hereditary family of finite subsets of $\mathbb{N}, \delta>0, M \in[\mathbb{N}]$ and $\xi<\omega_{1}$.

1. We shall say that $\mathfrak{F} \delta$-norms $\mathcal{A}$, if $\sup _{F \in \mathfrak{F}}|\mu|(F)>\delta$, for all $\mu \in \mathcal{A}$.

2. $\mathcal{A}$ is called $(\xi, M)$ large, if for every $L \in[M], \zeta<\xi$ and $\epsilon>0$ there exists $\mu \in \mathcal{A}$ such that $|\mu|(\mathbb{N} \backslash L)<\epsilon$ and $\|\mu\|_{\zeta}<\epsilon$.

We are now ready for the proof of the main result of this section. We first treat the case $n=1$.

Theorem 2.11. Let $\mathfrak{F}$ be a hereditary family of finite subsets of $\mathbb{N}, M \in[\mathbb{N}]$, $\xi<\omega_{1}$, and $\delta>0$. Assume that there exists $\mathcal{A} \subset \mathcal{M}$ which is $(\xi, M)$ large and $\delta$-normed by $\mathfrak{F}$. There exists $N \in[M]$ such that $S_{\xi}(N) \subset \mathfrak{F}$.

Proof. We first consider the case of $\xi$ being a successor ordinal, say $\xi=\zeta+1$. Let $P \in[M]$ and $m \in \mathbb{N}$. We claim that there exists $Q \in[P]$ such that

$$
\bigcup_{i=1}^{m} F_{i}^{\zeta}(L) \in \mathfrak{F} \text {, for all } L \in[Q] .
$$

Indeed, if this is not the case, we obtain through Lemma 2.9, $Q \in[P]$ such that

$$
\bigcup_{i=1}^{m} F_{i}^{\zeta}(L) \notin \mathfrak{F}, \text { for all } L \in[Q] .
$$

Since $\mathcal{A}$ is $(\xi, M)$ large, there exists $\mu \in \mathcal{A}$ such that

$$
\|\mu\|_{\zeta}<\frac{\epsilon}{m} \text { and }|\mu|(\mathbb{N} \backslash Q)<\frac{\epsilon}{m},
$$

where $0<\epsilon<\frac{\delta}{2}$. We also have that $\mathfrak{F} \delta$-norms $\mathcal{A}$ and therefore, there exists $F_{0} \in \mathfrak{F}$ such that $|\mu|\left(F_{0}\right)>\delta$. It follows that $|\mu|\left(F_{0} \bigcap Q\right)>\delta-\epsilon$, and thus, since $\mathfrak{F}$ is hereditary, we can assume that $F_{0} \subset Q$ and $|\mu|\left(F_{0}\right)>\delta-\epsilon$. Set

$$
L=F_{0} \cup\left\{q \in Q: q>\max F_{0}\right\} .
$$

Clearly, $L \in[Q]$. We may choose $k \in \mathbb{N}$ minimal with respect to $F_{0} \subset \bigcup_{i=1}^{k} F_{i}^{\zeta}(L)$. Now $k-1<m$, as $\bigcup_{i=1}^{k-1} F_{i}^{\zeta}(L) \subset F_{0}$ and so it belongs to $\mathfrak{F}$. Therefore, $k \leq m$ and 
thus

$$
\delta-\epsilon<|\mu|\left(F_{0}\right) \leq k\|\mu\|_{\zeta}<\epsilon \text {, by the choice of } \mu,
$$

contradicting the choice of $\epsilon$. Hence our claim holds and we can inductively choose

$$
\begin{gathered}
P_{1} \supset P_{2} \supset \cdots, \text { infinite subsets of } M \text { such that } \\
\bigcup_{i=1}^{n} F_{i}^{\zeta}(L) \in \mathfrak{F} \text {, for all } L \in\left[P_{n}\right] \text { and } n \in \mathbb{N} \text {. }
\end{gathered}
$$

Next choose $m_{1}<m_{2}<\cdots$ with $m_{n} \in P_{n}$, for all $n \in \mathbb{N}$. We set $N=\left(m_{n}\right)$ and claim that $S_{\xi}(N) \subset \mathfrak{F}$. Indeed, let $F \in S_{\xi}$ and assume that $\min F=n$. Then $F=\bigcup_{i=1}^{k} F_{i}$, where $F_{1}<\cdots<F_{k}$ belong to $S_{\zeta}$ and $k \leq n$. Applying Lemma 2.6 we obtain a finite sequence $\left(G_{i}\right)_{i=1}^{l}, l \leq k$, of consecutive maximal $S_{\zeta}$ subsets of $N$ with $\min G_{1}=m_{n}$ and such that

$$
\bigcup_{i=1}^{l-1} G_{i} \subset N(F) \subset \bigcup_{i=1}^{l} G_{i} .
$$

Note that $G_{i} \in\left[P_{n}\right]$, for $i \leq l$ and so there exists $L \in\left[P_{n}\right]$ such that

$$
F_{i}^{\zeta}(L)=G_{i}, \text { for all } i \leq l .
$$

We now obtain that $N(F) \in \mathfrak{F}$, as $l \leq n$. This completes the proof for the case of a successor ordinal $\xi$.

Now let $\xi$ be a limit ordinal and assume that the assertion of the theorem holds for all ordinals smaller than $\xi$. Let $\left(\xi_{n}+1\right)$ be the sequence of ordinals associated to $\xi$. We can now choose by the induction hypothesis,

$$
N_{1} \supset N_{2} \supset \cdots \text { infinite subsets of } M
$$

such that $S_{\xi_{n}+1}\left(N_{n}\right) \subset \mathfrak{F}$, for all $n \in \mathbb{N}$. Suppose that $N_{i}=\left(m_{k}^{i}\right)_{k \in \mathbb{N}}$, for all $i \in \mathbb{N}$, and choose $m_{1}<m_{2}<\cdots$ such that $m_{i} \in N_{i}$ and $m_{i}>m_{i}^{i}$, for all $i \in \mathbb{N}$. Set $N=\left(m_{i}\right)$ and it is easy to see that $S_{\xi}(N) \subset \mathfrak{F}$.

Notation. Let $s=\left(x_{n}\right)$ be a sequence in a Banach space $X$ and $\epsilon>0$. We set

$$
\mathfrak{F}_{\epsilon}(s)=\left\{F \in[\mathbb{N}]^{<\infty}: \exists x^{*} \in B_{X^{*}} \text { with }\left|x^{*}\left(x_{n}\right)\right| \geq \epsilon, \forall n \in F\right\} .
$$

Clearly, $\mathfrak{F}_{\epsilon}(s)$ is a hereditary family.

Corollary 2.12. Let $s=\left(x_{n}\right)$ be a sequence in a Banach space and $\delta>0$. Let $\xi$ be a countable ordinal and suppose that there exists a set $\mathcal{A}$ of absolutely sub-convex combinations of $\left(x_{n}\right)$ such that:

1. $\|x\|>\delta$, for all $x \in \mathcal{A}$.

2. For every $L \in[\mathbb{N}], \epsilon>0$ and $\zeta<\xi$, there exists $x \in \mathcal{A}, x=\sum_{i=1}^{\infty} a_{i} x_{i}$, such that $\sum_{i \notin L}\left|a_{i}\right|<\epsilon$ and $\sum_{i \in F}\left|a_{i}\right|<\epsilon$, for all $F \in S_{\zeta}$.

Then there exists $N \in[\mathbb{N}]$ such that $S_{\xi}(N) \subset \mathfrak{F}_{\frac{\delta}{2}}(s)$.

Theorem 2.13. Let $\mathfrak{F}$ be a hereditary family, $\xi<\omega_{1}, M \in[\mathbb{N}], \delta>0$ and $n \in \mathbb{N}$. Assume that there exists $\mathcal{A} \subset \mathcal{M}$, consisting of finitely supported measures, which is $(\xi, M)$ large. Assume further that if $\mu_{1}<\cdots<\mu_{n}$ and $\mu_{i} \in \mathcal{A}$, for $i \leq n$, then there exists $G \in \mathfrak{F}$ such that $\left|\mu_{i}\right|(G)>\delta$, for all $i \leq n$. Then, there exists $N \in[M]$ such that $S_{\xi}^{n}(M) \subset \mathfrak{F}$.

We shall need the following 
Lemma 2.14. Let $M \in[\mathbb{N}], n \in \mathbb{N}$ and $\xi<\omega_{1}$. Suppose that

$$
S_{\xi}^{n}(M)=\bigcup_{i=1}^{k} \mathfrak{F}_{i}
$$

where $\mathfrak{F}_{i}$ is hereditary for all $i \leq k$. Then there exist $N \in[M]$ and $i_{0} \leq k$ such that $S_{\xi}^{n}(N) \subset \mathfrak{F}_{i_{0}}$.

Proof. Define

$$
\Delta_{i}=\left\{L \in[\mathbb{N}]: M\left(\bigcup_{j=1}^{n} F_{j}^{\xi}(L)\right) \in \mathfrak{F}_{i}\right\}, \text { for } i \leq k .
$$

Evidently, $\Delta_{i}$ is closed in $[\mathbb{N}]$ and therefore Ramsey, for all $i \leq k$. Of course, by our assumption, we have that

$$
[\mathbb{N}]=\bigcup_{i=1}^{k} \Delta_{i} .
$$

We may now choose $P \in[\mathbb{N}]$ and $i_{0} \leq k$ such that $[P] \subset \Delta_{i_{0}}$. Let $N=M(P)$, and clearly $S_{\xi}^{n}(N) \subset \mathfrak{F}_{i_{0}}$.

Proof of Theorem 2.13. By induction on $n$. The case $n=1$ has been settled in Theorem 2.11. So assume $n \geq 2$. Choose $\mathcal{B}$ a countable dense subset of $\mathcal{A}$. Clearly, $\mathcal{B}$ satisfies the same assumptions as $\mathcal{A}$ does in the hypothesis of Theorem 2.13 Therefore, without loss of generality, we shall assume that $\mathcal{A}$ itself is countable. Let $\left(a_{k}\right)$ be an enumeration of the elements of $\mathcal{A}$. We claim that there exist:

1. A sequence $\left(G_{k}\right)$ of elements of $\mathfrak{F}$ such that $G_{k} \subset \operatorname{supp} a_{k}$ and $\left|a_{k}\right|\left(G_{k}\right)>\delta$, for all $k \in \mathbb{N}$.

2. A decreasing sequence $\left(M_{k}\right)$ of infinite subsets of $M$ such that supp $a_{k}<M_{k}$ and $G_{k} \cup F \in \mathfrak{F}$, for all $F \in S_{\xi}^{n-1}\left(M_{k}\right)$, and all $k \in \mathbb{N}$.

Indeed, suppose that $G_{1}, \cdots, G_{k-1}$ and $M_{1} \supset \cdots \supset M_{k-1}$ satisfying 1 and 2 have been constructed. Let $G \in \mathfrak{F}$ such that $G \subset \operatorname{supp} a_{k}$ and $\left|a_{k}\right|(G)>\delta$. Define

$$
\mathfrak{F}_{G}=\{F \in \mathfrak{F}: G<F \text { and } G \cup F \in \mathfrak{F}\} .
$$

Let $\Delta=\bigcup_{G} \mathfrak{F}_{G}$, where the union is taken over all possible subsets $G$ of $\operatorname{supp} a_{k}$ which belong to $\mathfrak{F}$ and satisfy the relation $\left|a_{k}\right|(G)>\delta$. Of course, $\Delta$ is hereditary and the hypothesis of Theorem 2.13 is satisfied for the family $\Delta$, the integer $n-1$ and the set of measures $\left\{a_{i}: a_{k}<a_{i}\right\}$. By the induction hypothesis there exists, for all $k \in \mathbb{N}, N_{k} \in\left[M_{k-1}\right]$ such that $\operatorname{supp} a_{k}<N_{k}$ and $S_{\xi}^{n-1}\left(N_{k}\right) \subset \Delta$. Next choose according to Lemma 2.14, $G_{k} \in \mathfrak{F}$ and $M_{k} \in\left[N_{k}\right]$ such that $G_{k} \subset \operatorname{supp} a_{k}$, $\left|a_{k}\right|\left(G_{k}\right)>\delta$ and $S_{\xi}^{n-1}\left(M_{k}\right) \subset \mathfrak{F}_{G_{k}}$. It follows now that $G_{k} \cup F \in \mathfrak{F}$, for all $F \in S_{\xi}^{n-1}\left(M_{k}\right)$. This completes the inductive construction and our claim holds.

Now let $F$ be a finite subset of $\mathbb{N}$ and set

$$
k_{F}=\min \left\{k \in \mathbb{N}: F \subset G_{k}\right\} .
$$

We let $k_{F}=0$, if $F$ is not contained in $G_{k}$, for all $k \in \mathbb{N}$. Inductively we construct a sequence of positive integers, $m_{1}<m_{2}<\cdots$, in the following manner: Suppose that $m_{1}<\cdots<m_{d}$ and $q_{1} \leq \cdots \leq q_{d-1}$ have been constructed. $\left(m_{1}\right.$ is chosen arbitrarily in $M_{1}$ and $q_{0}=0$.) We set

$$
q_{d}=\max \left\{k_{F}: F \subset\left\{m_{1}, \cdots, m_{d}\right\}\right\} \vee q_{d-1} .
$$


Next choose $m_{d+1} \in M_{q_{d}+1}$ such that $m_{d+1}>m_{d}$ and $m_{d+1}>m_{d+1}^{q_{d}+1}$, where $M_{i}=\left(m_{j}^{i}\right)_{j=1}^{\infty}$, for all $i \in \mathbb{N}$. Let $P=\left(m_{i}\right)$. We now claim that if $F \subset P$ is contained in $G_{k}$, for some $k \in \mathbb{N}$, then $F \cup H \in \mathfrak{F}$, for all $H \in S_{\xi}^{n-1}(P)$ such that $F<H$. Indeed, let $m_{d}=\max F$. Then $k_{F} \leq q_{d}$ and $F \subset G_{k_{F}}$. It suffices to show that $H \in S_{\xi}^{n-1}\left(M_{k_{F}}\right)$ for all $H \in S_{\xi}^{n-1}(P)$ such that $F<H$. Our claim will then follow by condition 2 above. To this end, let $H \in S_{\xi}^{n-1}(P)$, such that $F<H$. Suppose that $H=\left\{m_{i_{1}}, \cdots, m_{i_{t}}\right\}$, where $\left\{i_{1},<\cdots<, i_{t}\right\}$ belongs to $S_{\xi}^{n-1}$. Now, for all $r \leq t$,

$$
m_{i_{r}} \geq m_{d+1} \text { and thus } i_{r} \geq d+1
$$

So,

$$
m_{i_{r}}>m_{i_{r}}^{q_{i_{r}-1}} \geq m_{i_{r}}^{q_{d}} \geq m_{i_{r}}^{k_{F}}, \text { as } k_{F} \leq q_{d} .
$$

Hence, $H \in S_{\xi}^{n-1}\left(M_{k_{F}}\right)$, as claimed. Finally, consider the hereditary family

$$
\mathfrak{D}=\bigcup_{k=1}^{\infty}\left\{F: F \subset G_{k}\right\} .
$$

Clearly, the hypothesis of Theorem 2.11 is satisfied for $\mathfrak{D}$ and the set of measures $\left\{a_{k} \mid G_{k}: k \in \mathbb{N}\right\}$. We can thus find $N \in[P]$ such that $S_{\xi}(N) \subset \mathfrak{D}$. It is now easily verified that $S_{\xi}^{n}(N) \subset \mathfrak{F}$.

Our next proposition will enable us to verify that the set of measures

$$
\mathcal{A}_{\xi}=\left\{\xi_{n}^{L}: n \in \mathbb{N}, L \in[\mathbb{N}]\right\}
$$

is $(\xi, \mathbb{N})$ large, for all $\xi<\omega_{1}$.

Proposition 2.15. For every $M \in[\mathbb{N}], \epsilon>0$, and all ordinals $\zeta<\xi<\omega_{1}$, there exists $N \in[M]$ (depending on $M, \epsilon, \zeta, \xi$ ) such that $\left\|\xi_{n}^{L}\right\|_{\zeta}<\epsilon$, for all $L \in[N]$ and $n \in \mathbb{N}$.

Proof. It suffices to show the following:

Claim. Let $\xi<\omega_{1}$. For all $\zeta<\xi, \epsilon>0$ and $M \in[\mathbb{N}]$, there exists $L \in[M]$ such that $\left\|\xi_{1}^{L}\right\|_{\zeta}<\epsilon$.

Indeed, assuming our claim holds, we observe that the set $\left\{L \in[M]:\left\|\xi_{1}^{L}\right\|_{\zeta}<\epsilon\right\}$, is closed in $[M]$ and therefore Ramsey. Our claim now yields the existence of $N \in[M]$ such that $\left\|\xi_{1}^{L}\right\|_{\zeta}<\epsilon$, for every $L \in[N]$. By stability, we obtain the assertion of the proposition.

We shall prove our claim by transfinite induction on $\xi$. If $\xi=1$, then $\zeta=0$ and the claim is easily verified. Assuming our claim holds for all ordinals smaller than $\xi$, first let $\xi$ be a limit ordinal. Let $\left(\xi_{n}+1\right)$ be the sequence of ordinals associated to $\xi$. Suppose that $\zeta<\xi$ and choose $m \in M$ so that

$$
\zeta<\xi_{m} \text { and } \frac{1}{m}<\frac{\epsilon}{2} \text {. }
$$

We apply the induction hypothesis on the ordinal $\xi_{m}$ and the set $L_{1}=\{i \in M$ : $i \geq m\}$ to obtain $L_{2}, \cdots, L_{m}$, infinite subsets of $L_{1}$ such that

$$
\left[\xi_{m}\right]_{1}^{L_{1}}<\left[\xi_{m}\right]_{1}^{L_{2}}<\cdots<\left[\xi_{m}\right]_{1}^{L_{m}} \text { and }\left\|\left[\xi_{m}\right]_{1}^{L_{i}}\right\|_{\zeta}<\frac{\epsilon}{2 m}, \text { for } 2 \leq i \leq m .
$$


By stability property $P_{4}$, there exists $N \in[M]$ with $\min N=m$ and such that

$$
\left[\xi_{m}\right]_{i}^{N}=\left[\xi_{m}\right]_{1}^{L_{i}} \text {, for } i \leq m .
$$

Now,

$$
\left[\xi_{m}+1\right]_{1}^{N}=\frac{1}{m} \sum_{i=1}^{m}\left[\xi_{m}\right]_{i}^{N}=\xi_{1}^{N} .
$$

Hence,

$$
\left\|\xi_{1}^{N}\right\|_{\zeta} \leq \frac{1}{m}+\frac{m-1}{2 m} \epsilon<\epsilon .
$$

So our claim holds if $\xi$ is a limit ordinal.

Suppose now that $\xi=\alpha+1$. If $\zeta<\alpha$, choose according to the induction hypothesis $L_{1} \in[M]$ such that $\left\|\alpha_{1}^{L_{1}}\right\|_{\zeta}<\epsilon$. Let $m=\min \operatorname{supp} \alpha_{1}^{L_{1}}$ and choose again by the induction hypothesis, $L_{2}, \cdots, L_{m}$, infinite subsets of $M$ such that

$$
\alpha_{1}^{L_{1}}<\alpha_{1}^{L_{2}}<\cdots<\alpha_{1}^{L_{m}} \text { and }\left\|\alpha_{1}^{L_{i}}\right\|_{\zeta}<\epsilon \text {, for } 2 \leq i \leq m
$$

But once again, by stability, there exists $N \in[M]$ with $\min N=m$ and such that

$$
\alpha_{i}^{N}=\alpha_{1}^{L_{i}}, \text { for } i \leq m .
$$

Now, $\xi_{1}^{N}=\frac{1}{m} \sum_{i=1}^{m} \alpha_{i}^{N}$, and thus, $\left\|\xi_{1}^{N}\right\|_{\zeta}<\epsilon$.

The final case to consider is when $\zeta=\alpha$. Let $\left(\beta_{i}+1\right)$ be the sequence of ordinals associated to $\alpha$. Choose $m \in M$ such that $\frac{1}{m}<\frac{\epsilon}{2}$. Set $L_{1}=\{n \in M: n \geq m\}$. It follows that

$$
\left[\beta_{m}+1\right]_{1}^{L_{1}}=\alpha_{1}^{L_{1}} .
$$

Let $k_{1}=\max \operatorname{supp} \alpha_{1}^{L_{1}}$. Choose according to the induction hypothesis $L_{2} \in[M]$ with $k_{1}<\min L_{2}$ and such that

$$
\left\|\alpha_{1}^{L_{2}}\right\|_{\beta_{j}}<\frac{\epsilon}{2 k_{1}}, \text { for all } j \leq k_{1} .
$$

Let $k_{2}=\max \operatorname{supp} \alpha_{1}^{L_{2}}$. Successive repetitions of the previous argument yield

$$
\alpha_{1}^{L_{1}}<\alpha_{1}^{L_{2}}<\cdots<\alpha_{1}^{L_{m}} \text { with } L_{i} \in[M] \text { for } i \leq m,
$$

such that if $k_{i}=\max \operatorname{supp} \alpha_{1}^{L_{i}}$ for $i \leq m$, then

$$
\left\|\alpha_{1}^{L_{i}}\right\|_{\beta_{j}}<\frac{\epsilon}{2 k_{i-1}} \text {, for all } j \leq k_{i-1} \text { and } 2 \leq i \leq m \text {. }
$$

Stability now guarantees the existence of $L \in[M]$ with $\min L=m$ and such that

$$
\alpha_{i}^{L}=\alpha_{1}^{L_{i}}, \text { for all } i \leq m .
$$

Now, $\xi_{1}^{L}=\frac{1}{m} \sum_{i=1}^{m} \alpha_{i}^{L}$ and it remains to show that $\left\|\xi_{1}^{L}\right\|_{\alpha}<\epsilon$. Indeed, let $G \in S_{\alpha}$ and choose $i_{0} \leq m$ minimal with respect to $G \cap \operatorname{supp} \alpha_{i_{0}}^{L} \neq \emptyset$. Let $l=\min G$ and observe that $l \leq k_{i_{0}}$. Choose $p \leq l$ such that $G \in S_{\beta_{p}+1}$. There exist $q \leq l$ and $\left(G_{j}\right)_{j=1}^{q}$ consecutive members of $S_{\beta_{p}}$ such that $G=\bigcup_{j=1}^{q} G_{j}$. Note that

$$
q \leq l \leq k_{i_{0}} \text { and also } p \leq l \leq k_{i_{0}} .
$$

Thus,

$$
\left\|\alpha_{i}^{L}\right\|_{\beta_{p}}<\frac{\epsilon}{2 k_{i_{0}}}, \text { for } i_{0}<i \leq m \text {. }
$$


Therefore,

$$
\alpha_{i}^{L}(G) \leq q\left\|\alpha_{i}^{L}\right\|_{\beta_{p}}<k_{i_{0}} \frac{\epsilon}{2 k_{i_{0}}}=\frac{\epsilon}{2}, \text { for } i_{0}<i \leq m
$$

Hence,

$$
\xi_{1}^{L}(G)=\frac{1}{m}\left[\alpha_{i_{0}}^{L}(G)+\sum_{i=i_{0}+1}^{m} \alpha_{i}^{L}(G)\right]<\frac{1}{m}+\frac{\epsilon}{2}<\epsilon .
$$

and so $\left\|\xi_{1}^{L}\right\|_{\alpha}<\epsilon$. The proof of the claim is now complete.

We next recall the concept of the $(n, \xi, M, \delta)$ large families $[\mathrm{A}-\mathrm{M}-\mathrm{T}$, $\mathrm{A}-\mathrm{F}$.

Definition 2.16. Let $M \in[\mathbb{N}], \xi<\omega_{1}, \delta>0$ and $n \in \mathbb{N}$. The hereditary family $\mathfrak{F}$ is called $(n, \xi, M, \delta)$ large provided that for all $N \in[M]$ there exists $F \in \mathfrak{F}$ such that $\xi_{i}^{N}(F)>\delta$, for all $i \leq n$.

Corollary 2.17. Let $\mathfrak{F}$ be $(n, \xi, M, \delta)$ large. There exists $N \in[M]$ such that $S_{\xi}^{n}(N) \subset \mathfrak{F}$.

Proof. Proposition 2.15 and the fact that $\mathfrak{F}$ is $(n, \xi, M, \delta)$ large, immediately yield that $\mathfrak{F}$ and $\mathcal{A}_{\xi}$ satisfy the hypothesis of Theorem 2.13, The assertion of the corollary now follows.

\section{A generalization of Elton's theorem}

In the first part of this section we give the proof of Theorem [1.9 which extends Elton's nearly unconditional theorem [E, O1]. The second part of section 3 is devoted to the proofs of the results concerning the summability methods (Theorem 1.7), the convex unconditionality (Theorem 1.10) and the $S_{\xi}$ unconditionality (Theorem 1.11). We also discuss the duality between $c_{0}^{\xi}$ and $l_{1}^{\xi}$ spreading models (Theorem 1.12) as well as the $\xi$ Dunford-Pettis property (Corollary 1.13).

Let $s=\left(x_{n}\right)$ be a weakly null sequence in the Banach space $X$. Recall here that $s$ is additionally assumed to be a normalized, bimonotone, Schauder basic sequence. For this fixed sequence $s$ and the countable ordinal $\xi$, we have the following:

Definition 3.1. Let $M \in[\mathbb{N}], \lambda>0$ and $k \in \mathbb{N}$. The functional $x^{*} \in B_{X^{*}}$ is said to be $\xi$-good for $(\lambda, M, k)$, if $x^{*}\left(\xi_{i}^{M} \cdot s\right) \geq 0$, for all $i \leq k$, and $\sum_{i=1}^{k} x^{*}\left(\xi_{i}^{M} \cdot s\right)>\lambda$.

The main tool for proving Theorem [1.9] is the following combinatorial result.

Lemma 3.2. Let $\epsilon>0, \lambda>0$ and $N \in[\mathbb{N}]$. There exists $M \in[N]$ satisfying the following property: If $L \in[M], k \in \mathbb{N}$ and there exists $x^{*} \in B_{X^{*}}$ which is $\xi$-good for $(\lambda, L, k)$, then there exists $y^{*} \in B_{X^{*}}$ which is $\xi$-good for $(\lambda, L, k)$ and such that

$$
\sum_{n \in M \backslash \bigcup_{i=1}^{k} \operatorname{supp} \xi_{i}^{L}}\left|y^{*}\left(x_{n}\right)\right|<\epsilon .
$$

Proof. First choose $\left(\epsilon_{i}\right)_{i=0}^{\infty}$, a sequence of positive scalars such that $\sum_{i=0}^{\infty} \epsilon_{i}<\epsilon$. Let $T_{0}=\emptyset$ and $T_{n}=\{1, \cdots, n\}$, if $n \in \mathbb{N}$. By an $n$-tuple of positive integers $\left(m_{i}\right)_{i \in T_{n}}$, we shall either mean the empty tuple, if $n=0$, or, that $m_{1}<\cdots<m_{n}$, if $n \geq 1$. Now let $n \in \mathbb{N} \cup\{0\}$ and $F \subset T_{n}$. The $n$-tuple $\left(m_{i}\right)_{i \in T_{n}}$ and the infinite subset $L$ of $\mathbb{N},\left(L=\left(l_{i}\right)\right)$, are said to satisfy property $\left(F-E_{n}\right)$, provided that $m_{n}<l_{1}$, if $n \geq 1$, and the following statement holds: 
If $k \in \mathbb{N}$, and there exists $x^{*} \in B_{X^{*}}$ which is $\xi$-good for $\left(\lambda,\left\{m_{j}: j \in T_{n} \backslash F\right\} \cup\left\{l_{j}\right.\right.$ : $j \geq 2\}, k)$, then there exists $y^{*} \in B_{X^{*}}$ which is $\xi$-good for $\left(\lambda,\left\{m_{j}: j \in T_{n} \backslash F\right\} \cup\left\{l_{j}\right.\right.$ : $j \geq 2\}, k)$ and such that

$$
\sum_{j \in F}\left|y^{*}\left(x_{m_{j}}\right)\right|+\left|y^{*}\left(x_{l_{1}}\right)\right|<\sum_{i=0}^{n} \epsilon_{i} .
$$

Let us also say that $\left(m_{i}\right)_{i \in T_{n}}$ and $L$ satisfy property $\left(E_{n}\right)$, if they satisfy property $\left(F-E_{n}\right)$, for every $F \subset T_{n}$.

We shall inductively construct an increasing sequence $\left(m_{n}\right)_{n=1}^{\infty}$ of elements of $N$ and a decreasing sequence $\left(M_{n}\right)_{n=0}^{\infty}$ of infinite subsets of $N$ with $m_{n} \in M_{n-1}$, if $n \geq 1$, so that for every $n \in \mathbb{N} \cup\{0\}$, if $L \in\left[M_{n}\right]$, then $\left(m_{i}\right)_{i \in T_{n}}$ and $L$ satisfy property $\left(E_{n}\right)$.

The first inductive step is similar to the general one and therefore we shall not discuss it. Assume that $\left(m_{i}\right)_{i \in T_{n}}$ and $M_{0} \supset \cdots \supset M_{n}$, infinite subsets of $N$, with $m_{i} \in M_{i-1}$ for $1 \leq i \leq n$ have been constructed so that if $i \leq n$ and $L \in\left[M_{i}\right]$, then $\left(m_{j}\right)_{j \in T_{i}}$ and $L$ satisfy property $\left(E_{i}\right)$. Let $m_{n+1}=\min M_{n}$. Fix $F \subset T_{n+1}$ and define

$$
\Delta_{F}=\left\{L \in\left[M_{n}\right], L=\left(l_{i}\right):\left(m_{i}\right)_{i \in T_{n+1}} \text { and } L \text { satisfy }\left(F-E_{n+1}\right)\right\} .
$$

Clearly, $\Delta_{F}$ is closed in $\left[M_{n}\right]$ and therefore Ramsey. Suppose that for some $P \in$ $\left[M_{n}\right], P=\left(p_{i}\right)$, we had that $[P] \cap \Delta_{F}=\emptyset$. Let $q \in \mathbb{N}$ and set

$$
L_{j}=\left\{p_{j}\right\} \cup\left\{p_{i}: i>q\right\} \text {, for all } j \leq q .
$$

Since $L_{j} \notin \Delta_{F}$, for all $j \leq q$, there exist integers $\left(k_{j}\right)_{j=1}^{q}$ and functionals $\left(x_{j}^{*}\right)_{j=1}^{q}$ in $B_{X^{*}}$ so that letting $R=\left\{m_{i}: i \in T_{n+1} \backslash F\right\} \cup\left\{p_{i}: i>q\right\}$, we have that for all $j \leq q, x_{j}^{*}$ is $\xi$-good for $\left(\lambda, R, k_{j}\right)$; moreover, if $y^{*} \in B_{X^{*}}$ is $\xi$-good for $\left(\lambda, R, k_{j}\right)$, then

$$
\sum_{i \in F}\left|y^{*}\left(x_{m_{i}}\right)\right|+\left|y^{*}\left(x_{p_{j}}\right)\right| \geq \sum_{i=0}^{n+1} \epsilon_{i} .
$$

Next choose $j_{0} \leq q$ such that $k_{j_{0}}=\min \left\{k_{j}: j \leq q\right\}$. We observe that if $y^{*}$ is $\xi$-good for $\left(\lambda, R, k_{j_{0}}\right)$ and $y^{*}\left(x_{i}\right)=0$, for all $i>\max \operatorname{supp} \xi_{k_{j_{0}}}^{R}$, then $y^{*}$ is also $\xi$-good for $\left(\lambda, R, k_{j}\right)$, for every $j \leq q$.

Now let $t=\max F$ and note that

$$
R=\left\{m_{i}: i \in T_{t-1} \backslash F\right\} \cup\left\{m_{i}: t<i \leq n+1\right\} \cup\left\{p_{i}: i>q\right\} .
$$

By the induction hypothesis, since $\left(m_{i}\right)_{i \in T_{t-1}}$ and $\left\{m_{i}: t \leq i \leq n+1\right\} \cup\left\{p_{i}: i>q\right\}$ satisfy property $\left(E_{t-1}\right)$, there exists $z^{*} \in B_{X^{*}} \xi$-good for $\left(\lambda, R, k_{j_{0}}\right)$ and such that

$$
\sum_{i \in F \backslash\{t\}}\left|z^{*}\left(x_{m_{i}}\right)\right|+\left|z^{*}\left(x_{m_{t}}\right)\right|<\sum_{i=0}^{t-1} \epsilon_{i} .
$$

Thus,

$$
\sum_{i \in F}\left|z^{*}\left(x_{m_{i}}\right)\right|<\sum_{i=0}^{n} \epsilon_{i}
$$


Without loss of generality, since $\left(x_{i}\right)$ is bimonotone, we can assume that $z^{*}\left(x_{i}\right)=0$, for all $i>\max \operatorname{supp} \xi_{k_{j_{0}}}^{R}$. Our previous observation yields that $z^{*}$ is $\xi$-good for $\left(\lambda, R, k_{j}\right)$, for all $j \leq q$, and thus,

$$
\sum_{i \in F}\left|z^{*}\left(x_{m_{i}}\right)\right|+\left|z^{*}\left(x_{p_{j}}\right)\right| \geq \sum_{i=0}^{n+1} \epsilon_{i}, \text { for all } j \leq q .
$$

Hence, $\left|z^{*}\left(x_{p_{j}}\right)\right| \geq \epsilon_{n+1}$, for all $j \leq q$. We have reached a contradiction since $\left(x_{i}\right)$ is weakly null and $q \in \mathbb{N}$ was arbitrary. Concluding, there exists $L \in\left[M_{n}\right]$ such that $[L] \subset \Delta_{F}$. By repeating the previous argument successively over all possible subsets of $T_{n+1}$, we obtain $M_{n+1} \in\left[M_{n}\right]$ such that $\left[M_{n+1}\right] \subset \Delta_{F}$, for all $F \subset T_{n+1}$. This completes the inductive construction. Set $M=\left(m_{i}\right)$. Let $L \in[M]$ and $k \in \mathbb{N}$ and suppose that there exists $x^{*} \in B_{X^{*}}$ which is $\xi$-good for $(\lambda, L, k)$. Now let $m_{n}=\max \operatorname{supp} \xi_{k}^{L}$ and $F=\left\{i<n: m_{i} \notin \bigcup_{j=1}^{k} \operatorname{supp} \xi_{j}^{L}\right\}$. Our construction yields that $\left(m_{i}\right)_{i \in T_{n}}$ and $\left\{m_{i}: i \geq n+1\right\}$ satisfy $\left(F-E_{n}\right)$. We also have, by stability, that

$$
\xi_{j}^{L}=\xi_{j}^{R_{n}}, \text { for all } j \leq k,
$$

where $R_{n}=\left\{m_{i}: i \in T_{n} \backslash F\right\} \cup\left\{m_{i}: i>n+1\right\}$, and therefore there exists $y^{*} \in B_{X^{*}}$ which is $\xi$-good for $(\lambda, L, k)$ and such that

$$
\sum_{i \in F}\left|y^{*}\left(x_{m_{i}}\right)\right|<\sum_{i=0}^{n} \epsilon_{i}<\epsilon .
$$

But $\left(x_{i}\right)$ is bimonotone and thus we can assume that $y^{*}\left(x_{i}\right)=0$ for all $i>m_{n}$. Hence, $\sum_{i \in M \backslash \bigcup_{j=1}^{k} \operatorname{supp} \xi_{j}^{L}}\left|y^{*}\left(x_{i}\right)\right|<\epsilon$, as desired.

Proof of Theorem [1.9. Let $\delta>0$. Our goal is to find $M \in[\mathbb{N}]$ and a constant $C(\delta)>0$ such that if $L \in[M], n \in \mathbb{N}$, and $\left(a_{i}\right)_{i=1}^{n}$ are scalars in $[-1,1]$, then

$$
\left\|\sum_{i \in F} a_{i}\left(\xi_{i}^{L} \cdot s\right)\right\| \leq C(\delta) \max \left\{\delta,\left\|\sum_{i=1}^{n} a_{i}\left(\xi_{i}^{L} \cdot s\right)\right\|\right\},
$$

for all $F \subset\left\{i \leq n:\left|a_{i}\right| \geq \delta\right\}$. If this is accomplished, then a simple diagonalization argument yields $M \in[\mathbb{N}]$ which works for all $\delta>0$. We let $\lambda_{k}=1+\frac{k+1}{\delta}$, for all $k \in \mathbb{N} \cup\{0\}$. Inductively we construct a decreasing sequence $\left(M_{k}\right)_{k=0}^{\infty}$ of infinite subsets of $\mathbb{N}$ such that for all $k \in \mathbb{N} \cup\{0\}, M_{k}$ satisfies the conclusion of Lemma 3.2 for " $\lambda$ " $=\lambda_{k}$ and " $\epsilon$ " $=\delta$. Next choose $m_{1}<m_{2}<\cdots$ so that $m_{i} \in M_{i}$, for all $i \in \mathbb{N}$. Let $M=\left(m_{i}\right)$. We shall show that $M$ is desired. To this end, let $L \in[M]$ and scalars $\left(a_{i}\right)_{i=1}^{n}$ in $[-1,1]$. Let $F \subset\{1, \cdots, n\}$ such that $\left|a_{i}\right| \geq \delta$, for all $i \in F$. Choose $k \in \mathbb{N} \cup\{0\}$ so that

$$
k \leq\left\|\sum_{i=1}^{n} a_{i}\left(\xi_{i}^{L} \cdot s\right)\right\|<k+1
$$

and set $F_{k}=\{i \in F: i \geq k\}$. We claim that

$$
\left\|\sum_{i \in F_{k}} a_{i}\left(\xi_{i}^{L} \cdot s\right)\right\| \leq 4 \lambda_{k} .
$$


Assume this is not the case and choose $x^{*} \in B_{X^{*}}$ such that

$$
\left|\sum_{i \in F_{k}} a_{i} x^{*}\left(\xi_{i}^{L} \cdot s\right)\right|>4 \lambda_{k}
$$

We can further choose $G_{k} \subset F_{k}$ such that

$$
\left|\sum_{i \in G_{k}} a_{i} x^{*}\left(\xi_{i}^{L} \cdot s\right)\right|>\lambda_{k}
$$

the scalars $\left(a_{i}\right)_{i \in G_{k}}$ are all of the same sign, and, $x^{*}\left(\xi_{i}^{L} \cdot s\right) \geq 0$, for all $i \in G_{k}$, (by replacing $x^{*}$ by $-x^{*}$, if necessary). Observe that $\operatorname{supp} \xi_{i}^{L} \subset M_{k}$, when $k \leq i \leq n$, and thus by stability, there exists $y^{*} \in B_{X^{*}}$ such that

$$
\begin{gathered}
y^{*}\left(\xi_{i}^{L} \cdot s\right) \geq 0, \text { for all } i \in G_{k}, \\
\sum_{i \in G_{k}} y^{*}\left(\xi_{i}^{L} \cdot s\right)>\lambda_{k} \text { and } \sum_{i \in M_{k} \backslash \bigcup_{j \in G_{k}} \operatorname{supp} \xi_{j}^{L}}\left|y^{*}\left(x_{i}\right)\right|<\delta .
\end{gathered}
$$

But now,

$$
\begin{aligned}
\left|\sum_{i=k}^{n} a_{i} y^{*}\left(\xi_{i}^{L} \cdot s\right)\right| & \geq\left|\sum_{i \in G_{k}} a_{i} y^{*}\left(\xi_{i}^{L} \cdot s\right)\right|-\sum_{i \in\{k, \cdots, n\} \backslash G_{k}}\left|y^{*}\left(\xi_{i}^{L} \cdot s\right)\right| \\
& >\sum_{i \in G_{k}}\left|a_{i}\right| y^{*}\left(\xi_{i}^{L} \cdot s\right)-\sum_{i \in M_{k} \backslash \bigcup_{j \in G_{k}} \operatorname{supp} \xi_{j}^{L}}\left|y^{*}\left(x_{i}\right)\right| \\
& >\delta \lambda_{k}-\delta=k+1 .
\end{aligned}
$$

Thus, $\left\|\sum_{i=k}^{n} a_{i}\left(\xi_{i}^{L} \cdot s\right)\right\|>k+1$ and since $\left(x_{n}\right)$ is bimonotone, we also have that $\left\|\sum_{i=1}^{n} a_{i}\left(\xi_{i}^{L} \cdot s\right)\right\|>k+1$ which is a contradiction. Therefore, our claim holds and hence

$$
\left\|\sum_{i \in F} a_{i}\left(\xi_{i}^{L} \cdot s\right)\right\| \leq \begin{cases}4\left(1+\frac{1}{\delta}\right), & \text { if }\left\|\sum_{i=1}^{n} a_{i}\left(\xi_{i}^{L} \cdot s\right)\right\|<1 \\ k\left(5+\frac{8}{\delta}\right), & \text { if } k \leq\left\|\sum_{i=1}^{n} a_{i}\left(\xi_{i}^{L} \cdot s\right)\right\|<k+1, k \in \mathbb{N} .\end{cases}
$$

Concluding,

$$
\left\|\sum_{i \in F} a_{i}\left(\xi_{i}^{L} \cdot s\right)\right\| \leq C(\delta) \max \left\{\delta,\left\|\sum_{i=1}^{n} a_{i}\left(\xi_{i}^{L} \cdot s\right)\right\|\right\}
$$

where, $C(\delta)=\frac{1}{\delta}\left(5+\frac{8}{\delta}\right)$. The proof of Theorem 1.9 is now complete.

Remark. A refinement of the proof of Lemma 3.2 yields that given $\theta>0$ and $\epsilon>0$, there exists $M \in[\mathbb{N}]$ satisfying the following property: If $L \in[M], k \in \mathbb{N}$ and there exists $x^{*} \in B_{X^{*}}$ which is $\xi$-good for $(\lambda, L, k)$, then there exists $y^{*} \in B_{X^{*}}$ with $y^{*}\left(\xi_{i}^{L} \cdot s\right) \geq 0$ for $i \leq k$ and such that

$$
\begin{gathered}
\sum_{i=1}^{k} y^{*}\left(\xi_{i}^{L} \cdot s\right) \geq(1-\theta) \sum_{i=1}^{k} x^{*}\left(\xi_{i}^{L} \cdot s\right) \text { and } \\
\sum_{n \in M \backslash \bigcup_{i=1}^{k} \operatorname{supp} \xi_{i}^{L}}\left|y^{*}\left(x_{n}\right)\right|<\epsilon \sum_{i=1}^{k} y^{*}\left(\xi_{i}^{L} \cdot s\right) .
\end{gathered}
$$

This property in turn implies that the constant $C(\delta)$, found in the proof of Theorem 1.9, is actually proportional to $\frac{1}{\delta}$. 
Elton's aforementioned theorem follows immediately if we let $\xi=0$ in the statement of Theorem [1.9. As a corollary to Elton's theorem one obtains the following dichotomy [E], [O1]:

Corollary 3.3. A normalized weakly null sequence $\left(x_{n}\right)$ in a Banach space, either has a subsequence equivalent to the unit vector basis of $c_{0}$, or, a Schauder basic subsequence $\left(x_{m_{n}}\right)$ which is semi-boundedly complete. If the latter alternative occurs, then the sequence of the biorthogonal functionals to $\left(x_{m_{n}}\right)$, converges weakly to zero in the dual of the closed linear span of $\left(x_{m_{n}}\right)$.

Our next result is the dual version of Lemma 3.2.

Lemma 3.4. Let $s=\left(x_{n}\right)$ be a weakly null sequence in the Banach space $X$ and $\xi<\omega_{1}$. Given $\epsilon>0, \delta>0, N \in[\mathbb{N}]$, there exists $M \in[N]$ satisfying the following property: If $k \in \mathbb{N}, L \in[M]$, and there exists $x^{*} \in B_{X^{*}}$ so that $x^{*}\left(\xi_{n}^{L} \cdot s\right) \geq \delta$, for all $n \leq k$, then there exists $y^{*} \in B_{X^{*}}$ so that $y^{*}\left(\xi_{n}^{L} \cdot s\right) \geq \delta$, for all $n \leq k$, and,

$$
\sum_{n \in M \backslash \bigcup_{i=1}^{k} \operatorname{supp} \xi_{i}^{L}}\left|y^{*}\left(x_{n}\right)\right|<\epsilon .
$$

Proof. The proof is similar to that of Lemma 3.2. Let us now say that the functional $x^{*} \in B_{X^{*}}$ is good for $(\delta, L, k)$, if $x^{*}\left(\xi_{n}^{L} \cdot s\right) \geq \delta$, for all $n \leq k$. Next choose $\left(\epsilon_{i}\right)_{i=0}^{\infty}$, a sequence of positive scalars such that $\sum_{i=0}^{\infty} \epsilon_{i}<\epsilon$. Using the same notation and terminology as in Lemma 3.2 let $n \in \mathbb{N} \cup\{0\}$ and $F \subset T_{n}$. The $n$-tuple $\left(m_{i}\right)_{i \in T_{n}}$ and the infinite subset $L$ of $\mathbb{N},\left(L=\left(l_{i}\right)\right)$, are said to satisfy property $\left(F-E_{n}^{*}\right)$, provided that $m_{n}<l_{1}$, if $n \geq 1$, and the following statement holds:

If $k \in \mathbb{N}$, and there exists $x^{*} \in B_{X^{*}}$ which is good for $\left(\lambda,\left\{m_{j}: j \in T_{n} \backslash F\right\} \cup\left\{l_{j}\right.\right.$ : $j \geq 2\}, k)$, then there exists $y^{*} \in B_{X^{*}}$ which is good for $\left(\lambda,\left\{m_{j}: j \in T_{n} \backslash F\right\} \cup\left\{l_{j}\right.\right.$ : $j \geq 2\}, k)$ and such that

$$
\sum_{j \in F}\left|y^{*}\left(x_{m_{j}}\right)\right|+\left|y^{*}\left(x_{l_{1}}\right)\right|<\sum_{i=0}^{n} \epsilon_{i} .
$$

Let us also say that $\left(m_{i}\right)_{i \in T_{n}}$ and $L$ satisfy property $\left(E_{n}^{*}\right)$, if they satisfy property $\left(F-E_{n}^{*}\right)$, for every $F \subset T_{n}$.

We shall inductively construct an increasing sequence $\left(m_{n}\right)_{n=1}^{\infty}$ of elements of $N$, and a decreasing sequence $\left(M_{n}\right)_{n=0}^{\infty}$ of infinite subsets of $N$ with $m_{n} \in M_{n-1}$, if $n \geq 1$, so that for every $n \in \mathbb{N} \cup\{0\}$, if $L \in\left[M_{n}\right]$, then $\left(m_{i}\right)_{i \in T_{n}}$ and $L$ satisfy property $\left(E_{n}^{*}\right)$.

The first inductive step is similar to the general one and therefore we shall not discuss it. Assume that $\left(m_{i}\right)_{i \in T_{n}}$ and $M_{0} \supset \cdots \supset M_{n}$, infinite subsets of $N$, with $m_{i} \in M_{i-1}$ for $1 \leq i \leq n$ have been constructed so that if $i \leq n$ and $L \in\left[M_{i}\right]$, then $\left(m_{j}\right)_{j \in T_{i}}$ and $L$ satisfy property $\left(E_{i}^{*}\right)$. Let $m_{n+1}=\min M_{n}$. Fix $F \subset T_{n+1}$ and define

$$
\Delta_{F}=\left\{L \in\left[M_{n}\right], L=\left(l_{i}\right),:\left(m_{i}\right)_{i \in T_{n+1}} \text { and } L \text { satisfy }\left(F-E_{n+1}^{*}\right)\right\} .
$$

Clearly, $\Delta_{F}$ is closed in $\left[M_{n}\right]$ and therefore Ramsey. Arguing as we did in the proof of Lemma [3.2, we obtain $M_{n+1} \in\left[M_{n}\right]$ such that $\left[M_{n+1}\right] \subset \Delta_{F}$, for every $F \subset T_{n+1}$. Indeed, we need only modify the definition of $k_{j_{0}}$ in the argument of Lemma [3.2. We alternatively set $k_{j_{0}}=\max \left\{k_{j}: j \leq q\right\}$ and observe that if $y^{*}$ is good for $\left(\delta, R, k_{j_{0}}\right)$, then $y^{*}$ is also good for $\left(\delta, R, k_{j}\right)$, for every $j \leq q$. The argument of Lemma 3.2 is now carried over unaltered yielding the proof of Lemma 3.4 . 
In the proof of the Schreier unconditionality theorem [M-R, $\mathrm{O} 2$, we shall make use of the following:

Lemma 3.5. Let $\left(x_{n}\right)$ be a weakly null sequence in the Banach space $X$. Let $\epsilon>0$, $\delta>0$ and $k \in \mathbb{N}$. There exists $Q \in[\mathbb{N}], Q=\left(q_{n}\right)$, such that for every $x^{*} \in B_{X^{*}}$ and $F \in[\mathbb{N}]^{k}$ there exists $y^{*} \in B_{X^{*}}$ satisfying

$$
\sum_{i \in F}\left|x^{*}\left(x_{q_{i}}\right)-y^{*}\left(x_{q_{i}}\right)\right|<\delta \quad \text { and } \quad \sum_{i \notin F}\left|y^{*}\left(x_{q_{i}}\right)\right|<\epsilon .
$$

Proof. Once again, the proof is much similar to that of Lemma 3.2 First choose $\left(\epsilon_{i}\right)_{i=0}^{\infty}$, a sequence of positive scalars such that $\sum_{i=0}^{\infty} \epsilon_{i}<\epsilon$. Let $\vec{\lambda}=\left(\lambda_{1}, \cdots, \lambda_{k}\right)$ be an element of $[-1,1]^{k}$. We shall say that the functional $x^{*} \in B_{X^{*}}$ is $\vec{\lambda}$-good for $L$, where $L=\left(l_{i}\right)$ is an infinite subset of $\mathbb{N}$, if $\sum_{i=1}^{k}\left|x^{*}\left(x_{l_{i}}\right)-\lambda_{i}\right|<\delta$. Using the same notation and terminology as in Lemma 3.2, let $n \in \mathbb{N} \cup\{0\}$ and $F \subset T_{n}$. The $n$-tuple $\left(m_{i}\right)_{i \in T_{n}}$ and the infinite subset $L$ of $\mathbb{N},\left(L=\left(l_{i}\right)\right)$, are said to satisfy property $\left(F-O_{n}\right)$, provided that $m_{n}<l_{1}$, if $n \geq 1$, and the following statement holds:

If there exists $x^{*} \in B_{X^{*}}$ which is $\vec{\lambda}$ - $\operatorname{good}$ for $\left\{m_{j}: j \in T_{n} \backslash F\right\} \cup\left\{l_{j}: j \geq 2\right\}$, then there exists $y^{*} \in B_{X^{*}}$ which is $\vec{\lambda}$-good for $\left\{m_{j}: j \in T_{n} \backslash F\right\} \cup\left\{l_{j}: j \geq 2\right\}$ and such that

$$
\sum_{j \in F}\left|y^{*}\left(x_{m_{j}}\right)\right|+\left|y^{*}\left(x_{l_{1}}\right)\right|<\sum_{i=0}^{n} \epsilon_{i}
$$

Let us also say that $\left(m_{i}\right)_{i \in T_{n}}$ and $L$ satisfy property $\left(O_{n}\right)$, if they satisfy property $\left(F-O_{n}\right)$, for every $F \subset T_{n}$. We shall inductively construct an increasing sequence $\left(m_{n}\right)_{n=1}^{\infty}$ of elements of $\mathbb{N}$, and a decreasing sequence $\left(M_{n}\right)_{n=0}^{\infty}$ of infinite subsets of $\mathbb{N}$ with $m_{n} \in M_{n-1}$, if $n \geq 1$, so that for every $n \in \mathbb{N} \cup\{0\}$, if $L \in\left[M_{n}\right]$, then $\left(m_{i}\right)_{i \in T_{n}}$ and $L$ satisfy property $\left(O_{n}\right)$.

The first inductive step is similar to the general one and therefore we shall not discuss it. Assume that $\left(m_{i}\right)_{i \in T_{n}}$ and $M_{0} \supset \cdots \supset M_{n}$, infinite subsets of $N$, with $m_{i} \in M_{i-1}$ for $1 \leq i \leq n$ have been constructed so that if $i \leq n$ and $L \in\left[M_{i}\right]$, then $\left(m_{j}\right)_{j \in T_{i}}$ and $L$ satisfy property $\left(O_{i}\right)$. Let $m_{n+1}=\min M_{n}$. Fix $F \subset T_{n+1}$ and define

$$
\Delta_{F}=\left\{L \in\left[M_{n}\right], L=\left(l_{i}\right),:\left(m_{i}\right)_{i \in T_{n+1}} \text { and } L \text { satisfy }\left(F-O_{n+1}\right)\right\} .
$$

Clearly, $\Delta_{F}$ is closed in $\left[M_{n}\right]$ and therefore Ramsey. Arguing as we did in the proofs of Lemmas 3.2 and 3.4 we obtain $M_{n+1} \in\left[M_{n}\right]$ such that $\left[M_{n+1}\right] \subset \Delta_{F}$, for every $F \subset T_{n+1}$. The inductive construction is now complete and we set $M=$ $\left(m_{i}\right)$. It follows, by our construction, that if $F \in[\mathbb{N}]^{k}$ and $x^{*} \in B_{X^{*}}$ is $\vec{\lambda}$-good for $\left\{m_{j}: j \in F\right\} \cup\left\{m_{j}: j>\max F\right\}$, then there exists $y^{*} \in B_{X^{*}} \vec{\lambda}$-good for $\left\{m_{j}: j \in F\right\} \cup\left\{m_{j}: j>\max F\right\}$ and such that $\sum_{j \notin F}\left|y^{*}\left(x_{m_{j}}\right)\right|<\epsilon$. Let us then say that $M$ works for $\vec{\lambda}$. Finally, let $\mathcal{E}$ be a finite $\delta$-net in $[-1,1]^{k}$, and choose $Q \in[\mathbb{N}]$ which works for every $\vec{\lambda} \in \mathcal{E}$. It is easily verified that $Q$ is desired.

Lemma 3.4 provides an alternative proof of the fact that every normalized weakly null sequence admits a convexly unconditional subsequence.

Proof of Theorem 1.10, Let $\delta>0$. It suffices to find $M \in[\mathbb{N}], M=\left(m_{i}\right)$, so that if $F \in[\mathbb{N}]^{<\infty}$ and $\left(\lambda_{i}\right)_{i \in F}$ are scalars satisfying $\left\|\sum_{i \in F} \lambda_{i} x_{m_{i}}\right\|>\delta$ and $\sum_{i \in F}\left|\lambda_{i}\right| \leq 1$, 
then $\left\|\sum_{i=1}^{\infty} a_{i} x_{m_{i}}\right\|>\frac{\delta^{2}}{32}$, for all choices of scalars $\left(a_{i}\right)_{i=1}^{\infty} \subset c_{00}$ with $\max _{i}\left|a_{i}\right| \leq 1$ and such that $\left|a_{i}\right|=\left|\lambda_{i}\right|$, for all $i \in F$. Once this is accomplished, a simple diagonalization argument yields $M \in[\mathbb{N}]$ which works for all $\delta>0$. To this end, let $M=\left(m_{i}\right)$ be the infinite subset of $\mathbb{N}$ resulting from Lemma 3.4 applied on the sequence $\left(x_{n}\right)$ for $\xi=0, " \delta "=\frac{\delta}{2}$ and " $\epsilon "=\frac{\delta^{2}}{32}$. Now let $F \in[\mathbb{N}]<\infty$ and $\left(\lambda_{i}\right)_{i \in F}$ be scalars such that $\left\|\sum_{i \in F} \lambda_{i} x_{m_{i}}\right\|>\delta$ and $\sum_{i \in F}\left|\lambda_{i}\right| \leq 1$. Choose $x^{*} \in B_{X^{*}}$ such that $\left|\sum_{i \in F} \lambda_{i} x^{*}\left(x_{m_{i}}\right)\right|>\delta$ and set

$$
G=\left\{i \in F:\left|x^{*}\left(x_{m_{i}}\right)\right| \geq \frac{\delta}{2}\right\} .
$$

Clearly, $\left|\sum_{i \in G} \lambda_{i} x^{*}\left(x_{m_{i}}\right)\right|>\frac{\delta}{2}$. Next, let $\left(a_{i}\right)_{i=1}^{\infty} \subset c_{00}$ with $\max _{i}\left|a_{i}\right| \leq 1$ and $\left|a_{i}\right|=\left|\lambda_{i}\right|$, for all $i \in F$. By splitting $G$ into four sets in the obvious manner, we find $H \subset G$ such that

$$
\left|\sum_{i \in H} \lambda_{i} x^{*}\left(x_{m_{i}}\right)\right|>\frac{\delta}{8}
$$

the scalars $\left(\lambda_{i}\right)_{i \in H}$ are all of the same sign, and, $x^{*}\left(x_{m_{i}}\right) \geq \frac{\delta}{2}$ for all $i \in H$, (by replacing $x^{*}$ by $-x^{*}$, if necessary). Now choose $y^{*} \in B_{X^{*}}$ with $y^{*}\left(x_{m_{i}}\right) \geq \frac{\delta}{2}$, for all $i \in H$, and such that $\sum_{i \notin H}\left|y^{*}\left(x_{m_{i}}\right)\right|<\frac{\delta^{2}}{32}$. It follows that

$$
\begin{aligned}
\left\|\sum_{i=1}^{\infty} a_{i} x_{m_{i}}\right\| & \geq\left|\sum_{i \in H} a_{i} y^{*}\left(x_{m_{i}}\right)\right|-\sum_{i \notin H}\left|y^{*}\left(x_{m_{i}}\right)\right| \\
& >\sum_{i \in H}\left|\lambda_{i}\right| \frac{\delta}{2}-\frac{\delta^{2}}{32}>\frac{\delta^{2}}{32}
\end{aligned}
$$

Corollary 3.6. For a normalized weakly null sequence $s=\left(x_{n}\right)$ and $\xi<\omega_{1}$, the following are equivalent:

1. There exists $M \in[\mathbb{N}], M=\left(m_{i}\right)$, so that $\left(x_{m_{i}}\right)$ is an $l_{1}^{\xi}$ spreading model.

2. There exist $N \in[\mathbb{N}]$ and $\delta>0$ such that $S_{\xi}(N) \subset \mathfrak{F}_{\delta}(s)$.

Proof. Suppose first that 1 holds and let $C>0$ such that

$$
\left\|\sum_{i \in F} \lambda_{i} x_{m_{i}}\right\| \geq C \sum_{i \in F}\left|\lambda_{i}\right| \quad \text { for all } F \in S_{\xi}, \quad \text { and scalars }\left(\lambda_{i}\right)_{i \in F}
$$

Let $t$ denote the sequence $\left(y_{i}\right)$, where $y_{i}=x_{m_{i}}$, for all $i \in \mathbb{N}$. Then, $\left\|\xi_{1}^{L} \cdot t\right\| \geq C$, for all $L \in[\mathbb{N}]$. It follows that for $\delta=\frac{C}{2}$, the hereditary family

$$
\left\{F \in[\mathbb{N}]^{<\infty}: M(F) \in \mathfrak{F}_{\delta}(s)\right\}
$$

is $(1, \xi, \mathbb{N}, \delta)$ large. Corollary 2.17 now yields $N \in[M]$ so that $S_{\xi}(N) \subset \mathfrak{F}_{\delta}(s)$ and thus 2 holds.

Conversely, assume that 2 holds and choose $M=\left(m_{i}\right) \in[N]$ as in the proof of Theorem 1.10, applied on the sequence $\left(x_{n}\right)_{n \in N}$ for " $\delta$ " $=\frac{\delta}{4}$. Let $F \in S_{\xi}$ and scalars $\left(\lambda_{i}\right)_{i \in F}$ such that $\sum_{i \in F}\left|\lambda_{i}\right|=1$. We claim that $\left\|\sum_{i \in F} \lambda_{i} x_{m_{i}}\right\| \geq \frac{\delta^{2}}{512}$, 
which evidently yields 1 . Indeed, by our assumption, there exists $x^{*} \in B_{X^{*}}$ such that $\left|x^{*}\left(x_{m_{i}}\right)\right| \geq \delta$, for all $i \in F$. Next choose $G \subset F$ such that

$$
\sum_{i \in G}\left|\lambda_{i}\right| \geq \frac{1}{4}
$$

the scalars $\left(\lambda_{i}\right)_{i \in G}$ are all of the same sign, and, $x^{*}\left(x_{m_{i}}\right) \geq \delta$, for all $i \in G$, (by replacing $x^{*}$ by $-x^{*}$ if necessary). Therefore, $\left\|\sum_{i \in G} \lambda_{i} x_{m_{i}}\right\| \geq\left|\sum_{i \in G} \lambda_{i} x^{*}\left(x_{m_{i}}\right)\right| \geq$ $\frac{\delta}{4}$, and hence applying Theorem 1.10 we obtain that $\left\|\sum_{i \in F} \lambda_{i} x_{m_{i}}\right\| \geq \frac{1}{32}\left(\frac{\delta}{4}\right)^{2}=\frac{\delta^{2}}{512}$, as claimed.

Proof of Theorem 1.7. Assume that 2 does not hold. Let $M \in[\mathbb{N}]$ and $\epsilon>0$. It is easily seen that the set

$$
\mathcal{A}_{\epsilon}=\left\{L \in[M]:\left\|\xi_{1}^{L} \cdot s\right\|<\epsilon\right\}
$$

where $s=\left(x_{n}\right)$, is closed in $[M]$ and therefore Ramsey. If it were the case that $[P] \cap \mathcal{A}_{\epsilon}=\emptyset$, for some $P \in[M]$, then the family $\mathfrak{F}_{\frac{\epsilon}{2}}(s)$ would be $\left(1, \xi, P, \frac{\epsilon}{2}\right)$ large, and hence, by Corollaries 2.17 and 3.6. $\left(x_{n}\right)_{n \in L}$ would in turn be an $\ell_{1}^{\xi}$ spreading model, for some $L \in[P]$ contradicting our assumption. It follows now that we can construct $\left(M_{n}\right)$, a decreasing sequence of infinite subsets of $\mathbb{N}$, such that for all $n \in \mathbb{N}$,

$$
\left\|\xi_{1}^{L} \cdot s\right\|<\frac{1}{n}, \text { for all } L \in\left[M_{n}\right]
$$

Now let $N$ be any infinite subset of $M$ almost contained in each $M_{n}$, and it is easy to verify that $\left(x_{n}\right)$ is $(L, \xi)$ convergent, for all $L \in[N]$.

In order to show that 1 and 2 are mutually exclusive, assume that $\left(x_{m_{i}}\right)$ is an $\ell_{1}^{\xi}$ spreading model with constant $C$. Let $0<\epsilon<\frac{C}{1+C}$. We can choose $N \in[M]$ so that

$$
\begin{aligned}
F \backslash\{\min F\} \in S_{\xi}(M), & \text { for all } F \in S_{\xi}[N], \quad \text { (by Lemma [2.4), } \\
\left\|\xi_{1}^{L}\right\|_{0}<\epsilon, & \text { for all } L \in[N], \quad \text { (by Proposition 2.15). }
\end{aligned}
$$

It follows now that for every $L \in[N]$,

$$
\left\|\xi_{n}^{L} \cdot s\right\|>C-(1+C) \epsilon>0, \quad \text { for all } n \in \mathbb{N},
$$

and thus $\left(x_{n}\right)$ is not $(L, \xi)$ convergent. Hence 1 does not hold.

An immediate consequence of Theorem 1.7 is Corollary 1.8 ,

Proof of Corollary 1.8. Suppose that $s=\left(x_{n}\right)$ is an $\ell_{1}^{\xi}$ spreading model with constant $C$. It follows that $\left\|\xi_{n}^{L} \cdot s\right\| \geq C$, for all $n \in \mathbb{N}$ and $L \in[\mathbb{N}]$. Next, choose according to Theorem 1.7, $N \in[\mathbb{N}]$ so that $\left(x_{n}\right)$ is $(L, \xi+1)$ convergent, for every $L \in[N]$. Evidently, $\left(\xi_{n}^{L} \cdot s\right)$ is Cesaro summable for all $L \in[N]$.

We continue our applications of Theorem 1.10 with the following.

Proof of Theorem 1.11, Let $C$ be the $\ell_{1}^{\xi}$ spreading model constant of $\left(x_{n}\right)$. Let $M \in[\mathbb{N}], M=\left(m_{i}\right)$ satisfying the conclusion of Theorem 1.10 for the sequence $\left(x_{n}\right)$ and " $\delta "=\frac{C}{2}$. We claim that $\left(x_{m_{i}}\right)$ is $S_{\xi}$ unconditional. Indeed, let $n \in \mathbb{N}$ and scalars $\left(a_{i}\right)_{i=1}^{n}$ be given. Let $F \subset\{1, \cdots, n\}, F \in S_{\xi}$, such that $\left\|\sum_{i \in F} a_{i} x_{m_{i}}\right\|=1$. It follows that $C \sum_{i \in F}\left|a_{i}\right| \leq 1$ and $\left\|\sum_{i \in F} C a_{i} x_{m_{i}}\right\|>\frac{C}{2}$. If there exists $j \leq n$ so that $C\left|a_{j}\right|>1$, then, since $\left(x_{n}\right)$ is bimonotone, we obtain that $\left\|\sum_{i=1}^{n} a_{i} x_{m_{i}}\right\|>\frac{1}{C}$. 
So assuming that $C\left|a_{i}\right| \leq 1$, for all $i \leq n$, we obtain through Theorem 1.10 that $\left\|\sum_{i=1}^{n} a_{i} x_{m_{i}}\right\|>\frac{C}{128}$. Hence,

$$
\left\|\sum_{i \in F} a_{i} x_{m_{i}}\right\| \leq \frac{128}{C}\left\|\sum_{i=1}^{n} a_{i} x_{m_{i}}\right\|,
$$

for every $F \in S_{\xi}$ and all choices of scalars $\left(a_{i}\right)_{i=1}^{n}$.

We also obtain the result on Schreier unconditionality [M-R], $\mathrm{O} 2]$.

Corollary 3.7. Let $\left(x_{n}\right)$ be a normalized weakly null sequence in $X$ and $\epsilon>0$. There exists a subsequence $\left(x_{m_{i}}\right)$ of $\left(x_{n}\right)$ which is $2+\epsilon S_{1}$ unconditional.

Proof. First choose $\theta>0$ such that $(1+\theta)(2+\theta)<2+\epsilon$. By passing to a subsequence, if necessary, we can assume that $\left(x_{n}\right)$ is Schauder basic with basis constant $1+\theta$. We first show that for every $k \in \mathbb{N}$ and $N \in[\mathbb{N}]$, there exists $L \in[N], L=\left(l_{i}\right)$ so that

$$
\left\|\sum_{i \in F} a_{i} x_{l_{i}}\right\| \leq(1+\theta)\left\|\sum_{i=1}^{\infty} a_{i} x_{l_{i}}\right\|
$$

for every $F \in[\mathbb{N}]^{k}$ and all choices of scalars $\left(a_{i}\right)$ in $c_{00}$. Indeed, apply Lemma 3.5 to the sequence $\left(x_{n}\right)_{n \in N}$ to obtain $L \in[N], L=\left(l_{i}\right)$, satisfying the conclusion of that lemma for $k$, " $\delta "=\frac{\theta}{6}$, and " $\epsilon "=\frac{\theta}{6}$. Now let $\left(a_{i}\right) \subset c_{00}$ such that $\left\|\sum_{i=1}^{\infty} a_{i} x_{l_{i}}\right\|=1$. Let $F \in[\mathbb{N}]^{k}$ and choose $x^{*} \in B_{X^{*}}$ such that

$$
\left\|\sum_{i \in F} a_{i} x_{l_{i}}\right\|=\left|\sum_{i \in F} a_{i} x^{*}\left(x_{l_{i}}\right)\right| .
$$

Then choose $y^{*} \in B_{X^{*}}$ such that

$$
\sum_{i \in F}\left|x^{*}\left(x_{l_{i}}\right)-y^{*}\left(x_{l_{i}}\right)\right|<\frac{\theta}{6} \quad \text { and } \quad \sum_{i \notin F}\left|y^{*}\left(x_{l_{i}}\right)\right|<\frac{\theta}{6} .
$$

We now have the estimate

$$
\begin{aligned}
1= & \left\|\sum_{i=1}^{\infty} a_{i} x_{l_{i}}\right\| \geq\left|\sum_{i=1}^{\infty} a_{i} y^{*}\left(x_{l_{i}}\right)\right| \\
& \geq\left|\sum_{i \in F} a_{i} x^{*}\left(x_{l_{i}}\right)\right|-\sum_{i \in F}\left|a_{i}\right|\left|x^{*}\left(x_{l_{i}}\right)-y^{*}\left(x_{l_{i}}\right)\right|-\sum_{i \notin F}\left|a_{i}\right|\left|y^{*}\left(x_{l_{i}}\right)\right| \\
& \geq\left\|\sum_{i \in F} a_{i} x_{l_{i}}\right\|-3 \frac{\theta}{6}-3 \frac{\theta}{6},
\end{aligned}
$$

and thus, $\left\|\sum_{i \in F} a_{i} x_{l_{i}}\right\| \leq(1+\theta)\left\|\sum_{i=1}^{\infty} a_{i} x_{l_{i}}\right\|$, as desired. We can now choose a decreasing sequence $\left(M_{k}\right)$ of infinite subsets of $\mathbb{N}$ such that for every $k \in \mathbb{N}$,

$$
\left\|\sum_{m \in F} a_{m} x_{m}\right\| \leq(1+\theta)\left\|\sum_{m \in M_{k}} a_{m} x_{m}\right\|
$$

for all $F \in\left[M_{k}\right]^{j}$ and $j \leq k$, and all choices of scalars $\left(a_{m}\right)_{m \in M_{k}} \subset c_{00}$. Finally, choose $m_{1}<m_{2}<\cdots$ with $m_{i} \in M_{i}$, for all $i \in \mathbb{N}$, and set $M=\left(m_{i}\right)$. It is easily verified that the subsequence $\left(x_{m_{i}}\right)$ is $2+\epsilon S_{1}$ unconditional.

The final results of this section concern the duality between $c_{0} \xi$ and $\ell_{1}^{\xi}$ spreading models. We first recall the following: 
Definition 3.8. A sequence $\left(x_{n}\right)$ in a Banach space is called a $c_{0} \xi$ spreading model, $1 \leq \xi<\omega_{1}$, if there exists a constant $C>0$ such that

$$
\left\|\sum_{i \in F} a_{i} x_{i}\right\| \leq C \max _{i \in F}\left|a_{i}\right|
$$

for every $F \in S_{\xi}$ and all choices of scalars $\left(a_{i}\right)_{i \in F}$.

Proof of Theorem 1.12. Once again, we assume that our sequence $\left(x_{n}\right)$ is bimonotone. We can also assume, without loss of generality, that

$$
\sum_{i=1}^{\infty} \sum_{j \neq i}\left|x_{i}^{*}\left(x_{j}\right)\right|<\frac{\epsilon}{2} .
$$

Furthermore, we shall assume that $\left(x_{n}\right)$ satisfies the conclusion of Theorem 1.9 for $\xi=0$ and $M=\mathbb{N}$. That is, for all $\delta>0$ there exists a constant $C(\delta)>0$ such that for every $n \in \mathbb{N}$ and all scalars $\left(a_{i}\right)_{i=1}^{n}$ in $[-1,1]$, if $F \subset\{1, \cdots, n\}$ and $\left|a_{i}\right| \geq \delta$ for all $i \in F$, then

$$
\left\|\sum_{i \in F} a_{i} x_{i}\right\| \leq C(\delta)\left\|\sum_{i=1}^{n} a_{i} x_{i}\right\| .
$$

Assume first that 2 holds and choose $L \in[M], L=\left(l_{i}\right)$, and $C>0$ so that

$$
\left\|\sum_{i \in F} a_{i} x_{l_{i}}\right\| \leq C \max _{i \in F}\left|a_{i}\right|
$$

for every $F \in S_{\xi}$ and all choices of scalars $\left(a_{i}\right)_{i \in F}$. We claim that $\left(x_{l_{i}}^{*} \mid X_{M}\right)$ is an $\ell_{1}^{\xi}$ spreading model. Indeed, let $F \in S_{\xi}$ and scalars $\left(a_{i}\right)_{i=1}^{n}$ be given. For each $i \in F$, let $\epsilon_{i}$ be the sign of $a_{i} x_{l_{i}}^{*}\left(x_{l_{i}}\right)$. Of course, $\left\|\sum_{i \in F} \epsilon_{i} x_{l_{i}}\right\| \leq C$. Therefore,

$$
\begin{aligned}
C\left\|\sum_{i \in F} a_{i} x_{l_{i}}^{*} \mid X_{M}\right\| & \geq\left|\sum_{i \in F} a_{i} x_{l_{i}}^{*}\left(\sum_{j \in F} \epsilon_{j} x_{l_{j}}\right)\right| \\
& \geq \sum_{i \in F}\left|a_{i}\right|\left|x_{l_{i}}^{*}\left(x_{l_{i}}\right)\right|-\sum_{i \in F}\left|a_{i}\right| \sum_{j \neq i}\left|x_{l_{i}}^{*}\left(x_{l_{j}}\right)\right| \\
& \geq \frac{\epsilon}{2} \sum_{i \in F}\left|a_{i}\right|
\end{aligned}
$$

and thus 1 holds.

Conversely, assume that 1 holds. Choose, according to Corollary [3.6, $L \in[M]$, $L=\left(l_{i}\right)$ and $\delta>0$ such that

$$
S_{\xi}(L) \subset\left\{F \in[\mathbb{N}]^{<\infty}: \exists x^{* *} \in B_{X_{M}^{* *}} \text { with }\left|x^{* *}\left(x_{n}^{*} \mid X_{M}^{*}\right)\right| \geq \delta, \forall n \in F\right\} .
$$

It follows that

$$
S_{\xi}(L) \subset\left\{F \in[\mathbb{N}]^{<\infty}: \exists x \in B_{X_{M}} \text { with }\left|x_{n}^{*}(x)\right|>\frac{\delta}{2}, \forall n \in F\right\} .
$$

We now claim that for every $x \in X_{M}$, the sequence $\left(\frac{x_{m_{i}}^{*}(x)}{x_{m_{i}}^{*}\left(x_{m_{i}}\right)} x_{m_{i}}\right)$, where $M=$ $\left(m_{i}\right)$, is series bounded. Indeed, let $x=\sum_{i=1}^{\infty} c_{i} x_{m_{i}} \in B_{X_{M}}$ and note that by 
monotonicity we have that $\left|c_{i}\right| \leq 1$, for all $i \in \mathbb{N}$. Then, for all $k \in \mathbb{N}$,

$$
\begin{aligned}
\left\|\sum_{i=1}^{k} \frac{x_{m_{i}}^{*}(x)}{x_{m_{i}}^{*}\left(x_{m_{i}}\right)} x_{m_{i}}\right\| & \leq\left\|\sum_{i=1}^{k} c_{i} x_{m_{i}}\right\|+\left\|\sum_{i=1}^{k} \frac{1}{x_{m_{i}}^{*}\left(x_{m_{i}}\right)}\left(\sum_{j \neq i} c_{j} x_{m_{i}}^{*}\left(x_{m_{j}}\right)\right) x_{m_{i}}\right\| \\
& \leq\|x\|+\sum_{i=1}^{k} \frac{1}{\epsilon} \sum_{j \neq i}\left|x_{m_{i}}^{*}\left(x_{m_{j}}\right)\right|<\frac{3}{2}
\end{aligned}
$$

and our claim holds. Now let $F \in S_{\xi}$ and choose $x \in B_{X_{M}}$ such that $\left|x_{l_{i}}^{*}(x)\right|>\frac{\delta}{2}$, for all $i \in F$. Since $\left|\frac{x_{l_{i}}^{*}(x)}{x_{l_{i}}^{*}\left(x_{l_{i}}\right)}\right|>\frac{\delta}{2}$, for all $i \in F$, our initial assumptions on the sequence $\left(x_{n}\right)$ yield that

$$
\left\|\sum_{i \in F} \frac{x_{l_{i}}^{*}(x)}{x_{l_{i}}^{*}\left(x_{l_{i}}\right)} x_{l_{i}}\right\| \leq C\left(\frac{\delta \epsilon}{2}\right)\left\|\sum_{i=1}^{n} \frac{x_{m_{i}}^{*}(x)}{x_{m_{i}}^{*}\left(x_{m_{i}}\right)} x_{m_{i}}\right\|,
$$

where $m_{n}=l_{\max F}$. Thus, letting $b_{i}^{F}=\frac{x_{l_{i}}^{*}(x)}{x_{l_{i}}^{*}\left(x_{l_{i}}\right)}$, for $i \in F$, we have that

$$
\left\|\sum_{i \in F} b_{i}^{F} x_{l_{i}}\right\| \leq \frac{3}{2} C\left(\frac{\delta \epsilon}{2}\right)
$$

and $\frac{\delta}{2} \leq\left|b_{i}^{F}\right| \leq \frac{1}{\epsilon}$, for all $i \in F$ and $F \in S_{\xi}$. A standard argument now shows that

$$
\left\|\sum_{i \in F} a_{i} x_{l_{i}}\right\| \leq \frac{6}{\delta} C\left(\frac{\delta \epsilon}{2}\right) \max _{i \in F}\left|a_{i}\right|,
$$

for all $F \in S_{\xi}$ and scalars $\left(a_{i}\right)_{i \in F}$. Hence, 1 implies 2 .

Definition 3.9. The Banach space $X$ satisfies the $\xi$ Dunford-Pettis property ( $\xi$ $\mathrm{DP}), 1 \leq \xi<\omega_{1}$, if for every pair of weakly null sequences $\left(x_{n}\right)$ and $\left(x_{n}^{*}\right)$ in $X$ and $X^{*}$ respectively, with $\left(x_{n}^{*}\right) \xi$-convergent, we have that $\lim _{n} x_{n}^{*}\left(x_{n}\right)=0$.

$X$ is said to be hereditarily $\xi$-DP, if every subspace of $X$ satisfies the $\xi$-DP.

Proof of Corollary 1.13. Assume first that 1 holds. Let $Y$ be a subspace of $X$ and consider the normalized weakly null sequences $\left(x_{n}\right)$ and $\left(x_{n}^{*}\right)$ in $Y$ and $Y^{*}$ respectively, with $\left(x_{n}^{*}\right) \xi$-convergent. Suppose that for some $\epsilon>0$ and $M=\left(m_{i}\right) \epsilon$ $[\mathbb{N}]$, it was the case that $x_{m_{i}}^{*}\left(x_{m_{i}}\right)>\epsilon$, for all $i \in \mathbb{N}$. It follows that condition 2 of Theorem 1.12 is satisfied and therefore $\left(x_{m_{i}}^{*}\right)$ admits a subsequence which is an $\ell_{1}^{\xi}$ spreading model in $Y^{*}$. This contradicts with Theorem 1.7 as $\left(x_{n}^{*}\right)$ is $\xi$-convergent. Hence, $Y$ satisfies the $\xi$-DP and 2 holds.

Conversely, assume that 2 holds. Let $\left(x_{n}\right)$ be a normalized weakly null sequence in $X$ admitting no subsequence which is a $c_{0}^{\xi}$ spreading model. In particular, no subsequence of $\left(x_{n}\right)$ is equivalent to the unit vector basis of $c_{0}$, and thus by Corollary 3.3 there exists $M=\left(m_{i}\right) \in[\mathbb{N}]$ such that the sequence $\left(x_{m_{i}}^{*} \mid X_{M}\right)$ is weakly null in $X_{M}^{*}$ (we let $\left(x_{n}^{*}\right)$ denote the sequence of the biorthogonal functionals of $\left(x_{n}\right)$ ). Our assumption further yields that condition 2 of Theorem 1.12 fails for the space $X_{M}$ and the weakly null sequences $\left(x_{m_{i}}\right)$ and $\left(x_{m_{i}}^{*}\right) \mid X_{M}$ in $X_{M}$ and $X_{M}^{*}$ respectively. Thus condition 1 fails as well and so there exists $P=\left(p_{i}\right) \in[M]$ so that $x_{p_{i}}^{*} \mid X_{P}$ is $\xi$-convergent in $X_{P}^{*}$ according to Theorem 1.7 But $X_{P}$ is $\xi$-DP and thus, $1=$ $\lim _{i} x_{p_{i}}^{*}\left(x_{p_{i}}\right)=0$, which is absurd. 
Remark. Corollary 1.13 can be viewed as a partial generalization of Cembranos theorem [C], $\mathrm{K}-\mathrm{O}$, that a Banach space $X$ is hereditarily Dunford-Pettis if, and only if, every normalized weakly null sequence in $X$ admits a subsequence equivalent to the unit vector basis of $c_{0}$.

\section{Boundedly CONVEXly COMPLETE SEQUences}

This section is devoted to the proof of Theorem 1.5 which immediately yields Theorem 1.4. Our interest is concentrated in weakly null sequences without boundedly convexly complete subsequences. In the next series of lemmas, we describe their structure. We remark here that for the Schreier spaces $X_{\xi}, \xi<\omega$, described in Section 1, it can be shown that they contain no boundedly convexly complete sequences. However, an example of a weakly null sequence $\left(y_{n}\right)$ in $X_{\omega}$, is given in A-O, such that no convex block subsequence of $\left(y_{n}\right)$ satisfies the weak Banach-Saks property. It turns out that some subsequence of $\left(y_{n}\right)$, is boundedly convexly complete. We also mention that examples of boundedly convexly complete sequences can be constructed in $C\left(\omega^{\omega}\right)$, the Banach space of functions continuous on the ordinal interval $\left[1, \omega^{\omega}\right]$ endowed by the order topology.

In the sequel, $s=\left(x_{n}\right)$ will denote a normalized, bimonotone weakly null sequence in the Banach space $X$. We shall assume, without loss of generality, that $s$ satisfies the conclusion of Theorem 1.9, for $M=\mathbb{N}$ and $\xi=0$. That is, for every $\delta>0$, there exists $C(\delta)>0$ such that if $\left(a_{i}\right)_{i=1}^{n}$ are scalars in $[-1,1], n \in \mathbb{N}$, and $F \subset\{1, \cdots, n\}$ with $\left|a_{i}\right| \geq \delta$, for all $i \in F$, then $\left\|\sum_{i \in F} a_{i} x_{i}\right\| \leq C(\delta)\left\|\sum_{i=1}^{n} a_{i} x_{i}\right\|$.

Notation. $\quad$ 1. Given $t=\left(y_{i}\right)$, a sequence in a Banach space and $\mathbf{a}=\left(a_{i}\right)$, a scalar sequence, we let $\mathbf{a} \cdot t$ denote the sequence $\left(a_{i} y_{i}\right)$.

2. If $M \in[\mathbb{N}], M=\left(m_{i}\right)$, we let $t_{M}$ denote the sequence $\left(y_{m_{i}}\right)$.

3. Let $P=\left(p_{i}\right)$ and $Q=\left(q_{i}\right)$ be infinite subsets of $\mathbb{N}$ with $P$ almost contained in $Q$. Then $\mathbf{a}_{Q \mid P}=\left(c_{i}\right)$ is the scalar sequence obtained in the following manner: Set $c_{i}=0$, if $q_{i} \notin P$. Then set $c_{i}=a_{j}$, if $q_{i}=p_{j}$, for some $j \in \mathbb{N}$.

Lemma 4.1. Assume that $s=\left(x_{n}\right)$ has no subsequence which is b.c.c. There exist $M_{0} \in[\mathbb{N}]$ and $\delta_{0}>0$ such that for every $L \in\left[M_{0}\right]$ there exist a sequence of scalars $\left(a_{m}\right)_{m \in L}$ with $\left(a_{m} x_{m}\right)_{m \in L}$ series bounded by 1 , and a sequence $\left(F_{i}\right)_{i \in \mathbb{N}}$ of consecutive subsets of $L$ so that the following are satisfied for every $i \in \mathbb{N}$ :

$$
a_{m} \geq 0, \text { for all } m \in F_{i}, \sum_{m \in F_{i}} a_{m} \leq 1 \text {, and }\left\|\sum_{m \in F_{i}} a_{m} x_{m}\right\|>\delta_{0} .
$$

Proof. We first observe that if $P=\left(p_{i}\right)$ and $Q=\left(q_{i}\right)$ are infinite subsets of $\mathbb{N}$ with $P$ almost contained in $Q$, and $\mathbf{a}$ is a scalar sequence, then

$$
b\left(\mathbf{a}_{Q \mid P} \cdot s_{Q}\right) \leq b\left(\mathbf{a} \cdot s_{P}\right) \text {, and } \tau\left(\mathbf{a}, s_{P}\right) \leq \tau\left(\mathbf{a}_{Q \mid P}, s_{Q}\right) .
$$

To prove the lemma, it suffices to find $M_{0} \in[\mathbb{N}], \delta_{1}>0$ and $1 \leq K<\infty$, so that for every $L \in\left[M_{0}\right]$ there exists a scalar sequence a with $b\left(\mathbf{a} \cdot s_{L}\right) \leq K$ and $\tau\left(\mathbf{a}, s_{L}\right)>\delta_{1}$. Once this is accomplished, then $M_{0}$ and $\delta_{0}=\frac{\delta_{1}}{2 K}$ satisfy the conclusion of the lemma.

We now claim that there exist $N \in[\mathbb{N}]$ and $\delta_{1}>0$ such that for all $L \in[N]$ there exists a scalar sequence $\mathbf{a}$ with

$$
b\left(\mathbf{a} \cdot s_{L}\right)<\infty \text {, and } \tau\left(\mathbf{a}, s_{L}\right)>\delta_{1} .
$$


If this is not the case, we construct a decreasing sequence $\left(M_{i}\right)$ consisting of infinite subsets of $\mathbb{N}$ so that $\tau\left(\mathbf{a}, s_{M_{i}}\right) \leq \frac{1}{i}$, for every scalar sequence a with $b\left(\mathbf{a} \cdot s_{M_{i}}\right)<\infty$. Now let $M \in[\mathbb{N}]$ be almost contained in $M_{i}$, for all $i \in \mathbb{N}$, and choose a scalar sequence a such that

$$
b\left(\mathbf{a} \cdot s_{M}\right)<\infty \quad \text { and } \quad \tau\left(\mathbf{a}, s_{M}\right)>0 .
$$

It follows that

$$
b\left(\mathbf{a}_{M_{i} \mid M} \cdot s_{M_{i}}\right) \leq b\left(\mathbf{a} \cdot s_{M}\right)<\infty
$$

for all $i \in \mathbb{N}$ and thus,

$$
\tau\left(\mathbf{a}, s_{M}\right) \leq \tau\left(\mathbf{a}_{M_{i} \mid M}, s_{M_{i}}\right) \leq \frac{1}{i},
$$

for all $i \in \mathbb{N}$, which is absurd. Therefore our claim holds.

We next claim that there exist $M_{0} \in[N]$ and $K<\infty$ so that for every $L \in\left[M_{0}\right]$, there exists a scalar sequence a such that $b\left(\mathbf{a} \cdot s_{L}\right) \leq K$ and $\tau\left(\mathbf{a}, s_{L}\right)>\delta_{1}$. Were this claim false, we would choose a decreasing sequence $\left(N_{i}\right)$ of infinite subsets of $N$ such that for all $i \in \mathbb{N}$, if $\tau\left(\mathbf{a}, s_{N_{i}}\right)>\delta_{1}$, for some scalar sequence $\mathbf{a}$, then $b\left(\mathbf{a} \cdot s_{N_{i}}\right)>i$. Now let $T \in[N]$ be almost contained in each $N_{i}$ and choose a scalar sequence a such that

$$
b\left(\mathbf{a} \cdot s_{T}\right)<\infty \quad \text { and } \quad \tau\left(\mathbf{a}, s_{T}\right)>\delta_{1} .
$$

It follows that

$$
\tau\left(\mathbf{a}_{N_{i} \mid T}, s_{N_{i}}\right) \geq \tau\left(\mathbf{a}, s_{T}\right)>\delta_{1}
$$

for all $i \in \mathbb{N}$, and hence

$$
b\left(\mathbf{a} \cdot s_{T}\right) \geq b\left(\mathbf{a}_{N_{i} \mid T} \cdot s_{N_{i}}\right)>i
$$

for all $i \in \mathbb{N}$ which is absurd.

Remark. If $L \in\left[M_{0}\right],\left(F_{i}\right)_{i=1}^{\infty}$ and $\left(a_{m}\right)_{m \in L}$, are as in the conclusion of Lemma 4.1 then we shall call the sequence $\left(\sum_{m \in F_{i}} a_{m} x_{m}\right)_{i \in \mathbb{N}}$, a sub-convex block subsequence of $\left(x_{i}\right)$ supported by $L$ and satisfying the conclusion of Lemma 4.1

Lemma 4.2. Let $s=\left(x_{n}\right)$ be a weakly null sequence having no subsequence which is b.c.c. Let $M_{0} \in[\mathbb{N}]$ and $\delta_{0}>0$ satisfy the conclusion of Lemma 4.1] applied on s. Assume that $\alpha<\omega_{1}$ is a limit ordinal and denote by $\left(\alpha_{n}+1\right)$ the sequence of ordinals associated to $\alpha$. Suppose that for every $n \in \mathbb{N}$ and every $N \in\left[M_{0}\right]$ there exists $M \in[N]$ such that $\left(x_{m}\right)_{m \in M}$ is an $l_{1}^{\alpha_{n}+1}$ spreading model. Then, for every $N \in\left[M_{0}\right]$, there exists $M \in[N]$ such that $\left(x_{m}\right)_{m \in M}$ is an $l_{1}^{\alpha}$ spreading model.

Proof. Let $\mathcal{A}$ denote the set of those sub-convex combinations of the sequence $s$ which are members of a (not necessarily the same) sub-convex block subsequence of $s$ that satisfies the conclusion of Lemma 4.1.

We shall apply Corollary 2.12 to the sequence $s$ and the family $\mathcal{A}$ in order to obtain $T \in[N]$ so that $S_{\alpha}(T) \subset \mathfrak{F}_{\frac{\delta_{0}}{2}}(s)$. Corollary 3.6 will then yield that for some $M \in[T],\left(x_{m}\right)_{m \in M}$ is an $l_{1}^{\alpha}$ spreading model. To this end, it suffices to show that for every $i_{0} \in \mathbb{N}$, every $\epsilon>0$ and $L \in[N]$, there exists $x \in \mathcal{A}$ supported by $L$ and such that $\|x\|_{\alpha_{i_{0}}}<\epsilon$. Suppose this is not the case and choose according to the hypothesis $P \in[L]$ so that $\left(x_{m}\right)_{m \in P}$ is an $l_{1}^{\alpha_{i_{0}}+1}$ spreading model with constant $K$. Without loss of generality, by Theorem 1.11, we can assume that $\left(x_{m}\right)_{m \in P}$ is 
$S_{\alpha_{i_{0}}+1}$ unconditional with constant $C$. Next, choose by Lemma [2.4, $Q \in[P]$ such that if $F \in S_{\alpha_{i_{0}}+1}[Q]$, then $F \backslash\{\min F\} \in S_{\alpha_{i_{0}}+1}(P)$.

Since $s$ has no b.c.c. subsequence there exist, by Lemma 4.1, a sequence of scalars $\left(a_{q}\right)_{q \in Q}$ and a sequence $\left(F_{i}\right)_{i=1}^{\infty}$ of consecutive subsets of $Q$ with $\left(a_{q} x_{q}\right)_{q \in Q}$ series bounded by 1 , such that for all $i \in \mathbb{N}$,

$$
a_{q} \geq 0, \quad \text { for all } q \in F_{i}, \sum_{q \in F_{i}} a_{q} \leq 1 \quad \text { and } \quad\left\|\sum_{q \in F_{i}} a_{q} x_{q}\right\|>\delta_{0} .
$$

Note that $\sum_{q \in F_{i}} a_{q} x_{q} \in \mathcal{A}$, and moreover, it is supported by $L$ for all $i \in \mathbb{N}$. Thus, there exists, for every $i \in \mathbb{N}$,

$$
G_{i} \subset F_{i}, G_{i} \in S_{\alpha_{i_{0}}} \quad \text { so that } \sum_{q \in G_{i}} a_{q} \geq \epsilon .
$$

Next choose $p \in \mathbb{N}$ such that $(p-1) K \epsilon>C$. Choose also $j \in \mathbb{N}$ with $p<\min F_{j}$. Then

$$
\bigcup_{l=j+1}^{j+p} G_{l} \in S_{\alpha_{i_{0}}+1}[Q] \text { and thus } \bigcup_{l=j+2}^{j+p} G_{l} \in S_{\alpha_{i_{0}}+1}(P) .
$$

Therefore,

$$
\left\|\sum_{q \in \bigcup_{l=j+2}^{j+p} G_{l}} a_{q} x_{q}\right\| \geq K \sum_{q \in \bigcup_{l=j+2}^{j+p} G_{l}} a_{q} \geq K(p-1) \epsilon>C .
$$

However,

$$
\left\|\sum_{q \in \bigcup_{l=j+2}^{j+p} G_{l}} a_{q} x_{q}\right\| \leq C\left\|\sum_{q \leq \max G_{j+p}} a_{q} x_{q}\right\| \leq C .
$$

This contradiction completes the proof of the lemma.

Lemma 4.3. Let $s=\left(x_{n}\right)$ be a weakly null sequence having no subsequence which is b.c.c. There exist $M \in[\mathbb{N}], \xi<\omega_{1}$ and $\delta>0$ so that the following are satisfied:

1. $\left(x_{m}\right)_{m \in M}$ is an $\ell_{1}^{\xi}$ spreading model yet no subsequence of $\left(x_{m}\right)_{m \in M}$ is an $\ell_{1}^{\xi+1}$ spreading model.

2. For every $N \in[M]$, there exist $L \in[N]$ and a sequence of scalars $\left(a_{m}\right)_{m \in N}$ with $\left(a_{m} x_{m}\right)_{m \in N}$ series bounded by 1 so that for all $i \in \mathbb{N}$

$$
a_{m} \geq 0, \text { for all } m \in F_{i}^{\xi}(L), \sum_{m \in F_{i}^{\xi}(L)} a_{m} \leq 1 \quad \text { and }\left\|\sum_{m \in F_{i}^{\xi}(L)} a_{m} x_{m}\right\|>\delta \text {. }
$$

Proof. Let $M_{0} \in[\mathbb{N}]$ and $\delta_{0}>0$ satisfy the conclusion of Lemma 4.1 applied on $s$. We define

$$
\begin{array}{r}
\zeta=\min \left\{\alpha<\omega_{1}: \exists P \in\left[M_{0}\right] \text { such that } \forall L \in[P],\right. \\
\left.\left(x_{n}\right)_{n \in L} \text { is not an } \ell_{1}^{\alpha} \text { spreading model }\right\} .
\end{array}
$$

It follows by the results in $\mathrm{A}-\mathrm{A}$ that $\zeta$ is well defined. Moreover, Lemma 4.2 yields that $\zeta$ is not a limit ordinal and thus $\zeta=\xi+1$, for some countable ordinal $\xi$. Now choose $P \in\left[M_{0}\right]$ so that no subsequence of $\left(x_{n}\right)_{n \in P}$ is an $\ell_{1}^{\xi+1}$ spreading model. Since $\xi<\zeta$, there exists $Q \in[P]$ so that $\left(x_{n}\right)_{n \in Q}$ is an $\ell_{1}^{\xi}$ spreading model and of course no subsequence of $\left(x_{n}\right)_{n \in Q}$ is an $\ell_{1}^{\xi+1}$ spreading model. 
It follows now by Corollary [3.6, that for every $L \in[Q]$, the family $S_{\xi+1}(L)$ is not contained in $\mathfrak{F}_{\frac{\delta_{0}}{2}}(s)$. Using Lemma 2.9] as we did in the proof of Theorem[2.11, we find $M \in[Q]$ and $n_{0} \in \mathbb{N}$, so that

$$
\bigcup_{i=1}^{n_{0}} F_{i}^{\xi}(L) \notin \mathfrak{F}_{\frac{\delta_{0}}{2}}(s)
$$

for all $L \in[M]$. It follows now, by Lemma 2.6, that every member of the family $\mathfrak{F}_{\frac{\delta_{0}}{2}}(s)[M]$ can be expressed as the union of at most $n_{0}$ consecutive $S_{\xi}$ sets.

We are going to show that $M$ and $\delta=\frac{\delta_{0}}{2 n_{0}}$ satisfy 2 . Indeed, let $N \in[M]$ and choose

$$
n_{1}<A_{n_{1}}<n_{2}<A_{n_{2}}<\cdots
$$

so that for all $i \in \mathbb{N}, n_{i} \in N$ and $A_{n_{i}}$ is a maximal $S_{\xi}$ subset of $N$. Set $N_{1}=\left\{n_{i}\right.$ : $i \in \mathbb{N}\}$. By Lemma 4.1, there exist a sequence of scalars $\left(a_{m}\right)_{m \in N_{1}}$ and a sequence of consecutive subsets of $N_{1},\left(F_{i}\right)_{i=1}^{\infty}$ with $\left(a_{m} x_{m}\right)_{m \in N_{1}}$ series bounded by 1 , so that for every $i \in \mathbb{N}$

$$
a_{m} \geq 0, \quad \text { for all } m \in F_{i}, \sum_{m \in F_{i}} a_{m} \leq 1, \text { and }\left\|\sum_{m \in F_{i}} a_{m} x_{m}\right\|>\delta_{0} .
$$

We next choose, for every $i \in \mathbb{N}$, a functional $x_{i}^{*} \in B_{X^{*}}$ such that

$$
\left|\sum_{m \in F_{i}} a_{m} x_{i}^{*}\left(x_{m}\right)\right|>\delta_{0}
$$

and let

$$
G_{i}=\left\{m \in F_{i}:\left|x_{i}^{*}\left(x_{m}\right)\right| \geq \frac{\delta_{0}}{2}\right\} .
$$

It now follows, as $G_{i}$ belongs to $\mathfrak{F}_{\frac{\delta_{0}}{2}}(s)[M]$, that there exists, for every $i \in \mathbb{N}$,

$$
H_{i} \subset G_{i}, H_{i} \in S_{\xi}, \text { such that }\left|\sum_{m \in H_{i}} a_{m} x_{i}^{*}\left(x_{m}\right)\right|>\frac{\delta_{0}}{2 n_{0}}=\delta .
$$

By extending $H_{i}$ to a maximal $S_{\xi}$ subset of $H_{i} \cup A_{\max F_{i}}$, if necessary, we can assume without loss of generality, that $H_{i}$ itself is a maximal $S_{\xi}$ subset of $N$, for all $i \in \mathbb{N}$. We also extend the sequence $\left(a_{m}\right)_{m \in N_{1}}$ to a scalar sequence $\left(a_{m}\right)_{m \in N}$ in the obvious manner.

Concluding, there exist $H_{1}<H_{2}<\cdots$ maximal $S_{\xi}$ subsets of $N$ and a scalar sequence $\left(a_{m}\right)_{m \in N}$ with $\left(a_{m} x_{m}\right)_{m \in N}$ series bounded by 1 , so that for every $i \in \mathbb{N}$,

$$
a_{m} \geq 0, \quad \text { for all } m \in H_{i}, \sum_{m \in H_{i}} a_{m} \leq 1 \quad \text { and } \quad\left\|\sum_{m \in H_{i}} a_{m} x_{m}\right\|>\delta .
$$

Finally, choose $L \in[N]$ such that $F_{i}^{\xi}(L)=H_{i}$, for all $i \in \mathbb{N}$, and we are done.

Definition 4.4. Let $M \in[\mathbb{N}], L \in[M], n \in \mathbb{N}$ and $\delta>0$. The scalar sequence $\left(a_{m}\right)_{m \in M}$ is called $\xi$-faithful for $(\delta, L, n)$, provided the following conditions hold:

1. $\left|a_{m}\right| \leq \frac{\delta}{2}$, for all $m \in M$.

2. $\left(a_{m} x_{m}\right)_{m \in M}$ is series bounded by 1 . 
3. For every $i \leq n$ we have that

$$
a_{m} \geq 0, \quad \text { for all } m \in F_{i}^{\xi}(L), \sum_{m \in F_{i}^{\xi}(L)} a_{m} \leq 1 \quad \text { and }\left\|\sum_{m \in F_{i}^{\xi}(L)} a_{m} x_{m}\right\| \geq \delta .
$$

Lemma 4.5. Let $s=\left(x_{n}\right)$ have no subsequence which is b.c.c. Let $M \in[\mathbb{N}]$, $\xi<\omega_{1}$ and $\delta>0$ satisfy the conclusion of Lemma 4.3 applied on s.

1. If $\xi=0$, then some subsequence of $\left(x_{m}\right)_{m \in M}$ is equivalent to the unit vector basis of $c_{0}$.

2. If $\xi \geq 1$, then for every $N \in[M]$ there exists $P \in[N]$ so that for all $L \in[P]$ and $n \in \mathbb{N}$ there exists a scalar sequence $\left(a_{m}\right)_{m \in N}$ which is $\xi$-faithful for $(\delta, L, n)$.

Proof. If $\xi=0$, then according to Lemma 4.3 there exist a scalar sequence $\left(a_{m}\right)_{m \in M}$ and $L \in[M], L=\left(l_{i}\right)$, so that $\left(a_{m} x_{m}\right)_{m \in M}$ is series bounded by 1 and $a_{m}>\delta$, for all $m \in L$. It now follows that if $x^{*} \in B_{X^{*}}$, then for all $k \in \mathbb{N}$,

$$
\sum_{i=1}^{k}\left|x^{*}\left(x_{l_{i}}\right)\right| \leq \frac{2 C(\delta)}{\delta}
$$

and thus $\left(x_{l_{i}}\right)$ is equivalent to the unit vector basis of $c_{0}$.

Assume now that $\xi \geq 1$. Let $N \in[M]$ and $Q \in[N]$. It follows that no subsequence of $\left(x_{m}\right)_{m \in M}$ is equivalent to the unit vector basis of $c_{0}$ and therefore we obtain, by Corollary [3.3, $N_{0} \in[Q]$ such that $\left(x_{m}\right)_{m \in N_{0}}$ is semi-boundedly complete.

We next choose, according to Lemma 4.3, a scalar sequence $\left(a_{m}\right)_{m \in N_{0}}$ and $L_{0} \in$ $\left[N_{0}\right]$ with $\left(a_{m} x_{m}\right)_{m \in N_{0}}$ series bounded by 1 so that for all $i \in \mathbb{N}$,

$$
a_{m} \geq 0, \quad \text { for all } m \in F_{i}^{\xi}\left(L_{0}\right), \sum_{m \in F_{i}^{\xi}\left(L_{0}\right)} a_{m} \leq 1 \quad \text { and } \quad\left\|\sum_{m \in F_{i}^{\xi}\left(L_{0}\right)} a_{m} x_{m}\right\| \geq \delta \text {. }
$$

Since $\lim _{m \in N_{0}} a_{m}=0$, there exists $i_{0} \in \mathbb{N}$ such that $\left|a_{m}\right|<\frac{\delta}{2}$, for all $m \in N_{0}$, $m>\max F_{i_{0}}^{\xi}\left(L_{0}\right)$. Set

$$
N_{1}=\left\{m \in N_{0}: m>\max F_{i_{0}}^{\xi}\left(L_{0}\right)\right\} \quad \text { and } \quad L=\bigcup_{i>i_{0}} F_{i}^{\xi}\left(L_{0}\right) .
$$

We also extend the sequence $\left(a_{m}\right)_{m \in N_{1}}$ to a sequence $\left(a_{m}\right)_{m \in N}$ in the obvious manner. Evidently, $\left(a_{m}\right)_{m \in N}$ is $\xi$-faithful for $(\delta, L, n)$, for all $n \in \mathbb{N}$. We next consider the set

$$
\mathcal{D}=\left\{L \in[N]: \forall n \in \mathbb{N} \quad \exists\left(a_{m}\right)_{m \in N} \quad \xi \text {-faithful for }(\delta, L, n)\right\}
$$

which is of course closed in $[N]$ and therefore Ramsey. Our previous argument yields the existence of $P \in[N]$ such that $[P] \subset \mathcal{D}$. Clearly, $P$ satisfies the conclusion of part 2 of this lemma.

Lemma 4.6. Let $s=\left(x_{n}\right)$ be a weakly null sequence and assume that $\left(x_{n}^{*}\right)$, the sequence of functionals biorthogonal to $\left(x_{n}\right)$ is weakly null in $\left[\left(x_{n}\right)\right]^{*}$. Let $\xi<\omega_{1}$, $M \in[\mathbb{N}], \epsilon>0$ and $\delta>0$. There exists $N \in[M]$ satisfying the following property: If $L \in[N], n \in \mathbb{N}$ and there exists a scalar sequence $\left(a_{m}\right)_{m \in M}$ which is $\xi$-faithful 
for $(\delta, L, n)$, then there exists $\left(b_{m}\right)_{m \in M}$ which is $\xi$-faithful for $(\delta, L, n)$ and so that

$$
\sum_{m \in N \backslash \bigcup_{i=1}^{n} F_{i}^{\xi}(L)}\left|b_{m}\right|<\epsilon
$$

Proof. The proof is similar to those of Lemmas 3.2 and 3.4. First choose a sequence $\left(\epsilon_{i}\right)_{i=0}^{\infty}$ of positive scalars such that $\sum_{i=0}^{\infty} \epsilon_{i}<\epsilon$. Using the same notation and terminology as in Lemma 3.2, let $n \in \mathbb{N} \cup\{0\}$ and $F \subset T_{n}$. The $n$-tuple of elements of $M\left(r_{i}\right)_{i \in T_{n}}$ and the infinite subset $L$ of $M, L=\left(l_{i}\right)$, are said to satisfy property $\left(F-A_{n}\right)$ provided that $r_{n}<l_{1}$, if $n \geq 1$, and the following statement holds:

If $k \in \mathbb{N}$ and there exists a scalar sequence $\left(a_{m}\right)_{m \in M}$ which is $\xi$-faithful for $\left(\delta,\left\{r_{i}: i \in T_{n} \backslash F\right\} \cup\left\{l_{i}: i \geq 2\right\}, k\right)$, then there exists $\left(b_{m}\right)_{m \in M}$ which is $\xi$-faithful for $\left(\delta,\left\{r_{i}: i \in T_{n} \backslash F\right\} \cup\left\{l_{i}: i \geq 2\right\}, k\right)$ and such that

$$
\sum_{i \in F}\left|b_{r_{i}}\right|+\left|b_{l_{1}}\right|<\sum_{i=0}^{n} \epsilon_{i}
$$

Let us also say that $\left(r_{i}\right)_{i \in T_{n}}$ and $L$ satisfy property $\left(A_{n}\right)$, if they satisfy property $\left(F-A_{n}\right)$, for all $F \subset T_{n}$.

We shall inductively construct an increasing sequence $\left(r_{n}\right)_{n=1}^{\infty}$ of elements of $M$ and a decreasing sequence $\left(M_{n}\right)_{n=0}^{\infty}$ of infinite subsets of $M$ with $r_{n} \in M_{n-1}$, if $n \geq 1$, so that if $n \in \mathbb{N} \cup\{0\}$ and $L \in\left[M_{n}\right]$, then $\left(r_{i}\right)_{i \in T_{n}}$ and $L$ satisfy property $\left(A_{n}\right)$.

The first inductive step is similar to the general one and so we shall not discuss it. Now we assume that $r_{1}<\cdots<r_{n}$ and $M_{0} \supset \cdots \supset M_{n}$ have been constructed with $r_{i} \in M_{i-1}$, if $i \geq 1$, so that if $i \leq n$, and $L \in\left[M_{i}\right]$, then $\left(r_{j}\right)_{j \in T_{i}}$ and $L$ satisfy property $\left(A_{i}\right)$. Let $r_{n+1}=\min M_{n}$ and fix $F \subset T_{n+1}$. We define

$$
\Delta_{F}=\left\{L \in\left[M_{n}\right]:\left(r_{i}\right)_{i \in T_{n+1}} \text { and } L \text { satisfy }\left(F-A_{n+1}\right)\right\} .
$$

Clearly, $\Delta_{F}$ is closed in $\left[M_{n}\right]$ and therefore Ramsey. Suppose that for some $P \in$ $\left[M_{n}\right], P=\left(p_{i}\right)$, we had that $[P] \cap \Delta_{F}=\emptyset$. Let $q \in \mathbb{N}$ and set

$$
L_{j}=\left\{p_{j}\right\} \cup\left\{p_{i}: i>q\right\}, \text { for all } j \leq q .
$$

Since $L_{j} \notin \Delta_{F}$, for all $j \leq q$, there exist integers $\left(k_{j}\right)_{j=1}^{q}$ as well as scalar sequences $\left(a_{m}^{j}\right)_{m \in M}, j \leq q$, so that letting $R=\left\{r_{i}: i \in T_{n+1} \backslash F\right\} \cup\left\{p_{i}: i>q\right\}$ we have that for all $j \leq q$,

$$
\left(a_{m}^{j}\right)_{m \in M} \text { is } \xi \text {-faithful for }\left(\delta, R, k_{j}\right),
$$

and moreover, if $\left(b_{m}\right)_{m \in M}$ is a $\xi$-faithful scalar sequence for $\left(\delta, R, k_{j}\right)$, then

$$
\sum_{i \in F}\left|b_{r_{i}}\right|+\left|b_{p_{j}}\right| \geq \sum_{i=0}^{n+1} \epsilon_{i} .
$$

Let $k_{0}=\max \left\{k_{j}: j \leq q\right\}$ and observe that any scalar sequence $\left(b_{m}\right)_{m \in M}$ which is $\xi$-faithful for $\left(\delta, R, k_{0}\right)$, is also $\xi$-faithful for $\left(\delta, R, k_{j}\right)$, for all $j \leq q$. Next, let $t=\max F$ and note that

$$
R=\left\{r_{i}: i \in T_{t-1} \backslash F\right\} \cup\left\{r_{i}: t<i \leq n+1\right\} \cup\left\{p_{i}: i>q\right\} .
$$

By the induction hypothesis, since $\left(r_{i}\right)_{i=1}^{t-1}$ and $\left\{r_{i}: t \leq i \leq n+1\right\} \cup\left\{p_{i}: i>q\right\}$ satisfy property $\left(A_{t-1}\right)$, there exists a scalar sequence $\left(b_{m}\right)_{m \in M}$ which is $\xi$-faithful 
for $\left(\delta, R, k_{0}\right)$ and such that

$$
\sum_{i \in F \backslash\{t\}}\left|b_{r_{i}}\right|+\left|b_{r_{t}}\right|<\sum_{i=0}^{t-1} \epsilon_{i} .
$$

Thus,

$$
\sum_{i \in F}\left|b_{r_{i}}\right|<\sum_{i=0}^{n} \epsilon_{i}
$$

It follows now by our previous observation, that $\left(b_{m}\right)_{m \in M}$ is $\xi$-faithful for $\left(\delta, R, k_{j}\right)$, for all $j \leq q$ and thus,

$$
\sum_{i \in F}\left|b_{r_{i}}\right|+\left|b_{p_{j}}\right| \geq \sum_{i=0}^{n+1} \epsilon_{i}, \text { for all } j \leq q .
$$

Hence, $\left|b_{p_{j}}\right| \geq \epsilon_{n+1}$, for all $j \leq q$.

Now choose $m_{0}>p_{q}, m_{0} \in M$. Then, $\left|x_{p_{j}}^{*}\left(\sum_{m<m_{0}} b_{m} x_{m}\right)\right| \geq \epsilon_{n+1}$, for all $j \leq q$. But $\left\|\sum_{m \leq m_{0}} b_{m} x_{m}\right\| \leq 1$ and $q$ is arbitrary and so $\left(x_{p_{i}}^{*}\right)$ is not weakly null in $\left[\left(x_{i}\right)\right]^{*}$, contradicting our assumption.

Concluding, there exists $L \in\left[M_{n}\right]$ such that $[L] \subset \Delta_{F}$. By repeating the previous argument successively over all possible subsets of $T_{n+1}$, we obtain $M_{n+1} \in\left[M_{n}\right]$ such that $\left[M_{n+1}\right] \subset \Delta_{F}$, for all $F \subset T_{n+1}$. The inductive construction is now complete. Set $N=\left(r_{i}\right)$. Let $L \in[N]$ and $k \in \mathbb{N}$. Let $\left(a_{m}\right)_{m \in M}$ be a scalar sequence which is $\xi$-faithful for $(\delta, L, k)$. Suppose that $r_{p}=\max F_{k}^{\xi}(L)$ and let $G_{q}=\left\{i \in T_{q}: r_{i} \notin \bigcup_{j=1}^{k} F_{j}^{\xi}(L)\right\}$, for all $q \geq p$. Our construction yields that $\left(r_{i}\right)_{i=1}^{q}$ and $\left\{r_{i}: i>q\right\}$, satisfy $\left(G_{q}-A_{q}\right)$, for all $q \geq p$. We also have, by stability, that for all $q \geq p$,

$$
F_{j}^{\xi}(L)=F_{j}^{\xi}\left(R_{q}\right), \text { for all } j \leq k
$$

where, $R_{q}=\left\{r_{i}: i \in T_{q} \backslash G_{q}\right\} \cup\left\{r_{i}: i>q+1\right\}$, and therefore there exists a scalar sequence $\left(b_{m}^{q}\right)_{m \in M}$ which is $\xi$-faithful for $(\delta, L, k)$ and such that

$$
\sum_{i \in G_{q}}\left|b_{r_{i}}^{q}\right|<\sum_{i=0}^{q} \epsilon_{i}<\epsilon .
$$

Thus,

$$
\sum_{m \in N \backslash \bigcup_{i=1}^{k} F_{i}^{\xi}(L), m \leq r_{q}}\left|b_{m}^{q}\right|<\epsilon .
$$

Finally, let $\left(b_{m}\right)_{m \in M}$ be any cluster point of the sequence $\left(\left(b_{m}^{q}\right)_{m \in M}\right)_{q \geq p}$ in $[-1,1]^{M}$. Evidently, this is the desired sequence.

Lemma 4.7. Let $t=\left(y_{i}\right)$ be a sequence in $B_{X}, \xi<\omega_{1}$ and $\mu$ a finitely supported probability measure on $\mathbb{N}$. Assume there exist $x^{*} \in B_{X^{*}}$ and $\epsilon>0$ so that $x^{*}(\mu \cdot t) \geq$ $2 \epsilon$. Assume further that there exists $l \in \mathbb{N}, l<\operatorname{supp} \mu$, such that $l\|\mu\|_{\zeta_{i}}<\epsilon$, for every $i \leq l$, where $\left(\zeta_{i}+1\right)$ is the sequence of ordinals associated to $\xi$. Let $E=\left\{n \in \operatorname{supp} \mu: x^{*}\left(y_{n}\right) \geq \frac{1}{2} x^{*}(\mu \cdot t)\right\}$. Then, there exists a maximal $S_{\xi}$ set containing $l$ and contained in $\{l\} \cup E$. 
Proof. Assume on the contrary, that no subset of $\{l\} \cup E$ containing $l$ is a maximal $S_{\xi}$ set. We then claim that $\{l\} \cup E$ belongs to $S_{\xi}$. Indeed, suppose that $\{l\} \cup E=$ $\left\{m_{1}, \cdots, m_{k}\right\}, m_{1}=l$, and choose $r \leq k$ maximal with respect to $\left\{m_{1}, \cdots, m_{k}\right\} \in$ $S_{\xi}$. If $r<k$, then Lemma 2.5 yields that $\left\{m_{1}, \cdots, m_{r}\right\}$ is a maximal $S_{\xi}$ set contradicting our assumption. Thus $r=k$ as claimed.

There exists now $i \leq l$ such that $\{l\} \cup E$ belongs to $S_{\zeta_{i}+1}$. It follows that we can find $p \leq l$ and consecutive $S_{\zeta_{i}}$ sets $\left(A_{j}\right)_{j=1}^{p}$ so that

$$
\{l\} \cup E=\bigcup_{j=1}^{p} A_{j} .
$$

But now,

$$
\begin{aligned}
x^{*}(\mu \cdot t)= & \sum_{n \in E} \mu(n) x^{*}\left(y_{n}\right)+\sum_{n \in \operatorname{supp} \mu \backslash E} \mu(n) x^{*}\left(y_{n}\right) \\
& \leq \sum_{j=1}^{p} \mu\left(A_{j}\right)+\frac{1}{2} x^{*}(\mu \cdot t) \\
& \leq p\|\mu\|_{\zeta_{i}}+\frac{1}{2} x^{*}(\mu \cdot t)
\end{aligned}
$$

and thus $x^{*}(\mu \cdot t)<2 \epsilon$. This contradiction completes the proof of the lemma.

We are now ready for the proof of the main result of this paper.

Proof of Theorem 1.5. Assume that $s$ has no subsequence which is b.c.c. Choose $M \in[\mathbb{N}], \xi<\omega_{1}$ and $\delta>0$ satisfying the conclusion of Lemma 4.5 applied on $s$. If $\xi=0$, we are done since some subsequence of $s$ is equivalent to the unit vector basis of $c_{0}$.

Assume now that $\xi \geq 1$. Choose according to Corollary $3.3, M_{1} \in[M]$ so that the sequence $\left(x_{m}^{*}\right)_{m \in M_{1}}$ of functionals biorthogonal to $\left(x_{m}\right)_{m \in M_{1}}$ is weakly null in $\left[\left(x_{m}\right)_{m \in M_{1}}\right]^{*}$. Next, choose according to Lemma $2.4 M_{2} \in\left[M_{1}\right]$ such that

$$
F \backslash\{\min F\} \in S_{\xi}\left(M_{1}\right) \text {, for all } F \in S_{\xi}\left[M_{2}\right] .
$$

Let $0<\epsilon<1$ and choose a sequence of positive scalars $\left(\epsilon_{n}\right)$ such that $\sum_{n=1}^{\infty} \epsilon_{n}<\epsilon$. Choose also $\lambda \geq \frac{4}{\delta}(1+4 \epsilon)$. Lemma 3.2 now yields $M_{3} \in\left[M_{2}\right]$ such that for every $L \in\left[M_{3}\right]$ and $n \in \mathbb{N}$, if there exists $x^{*} \in B_{X^{*}}$ which is $\xi$-good for $(\delta, L, n)$, then there exists $y^{*} \in B_{X^{*}}, \xi$-good for $(\delta, L, n)$ and such that

$$
\sum_{m \in M_{3} \backslash \bigcup_{i=1}^{n} \operatorname{supp} \xi_{i}^{L}}\left|y^{*}\left(x_{m}\right)\right|<\epsilon .
$$

We continue our choice of infinite subsets of $M$ by choosing $M_{4} \in\left[M_{3}\right]$ according to Lemma 4.6. Thus, for every $L \in\left[M_{4}\right]$ and $n \in \mathbb{N}$, if there exists a scalar sequence $\left(a_{m}\right)_{m \in M_{3}}$ which is $\xi$-faithful for $(\delta, L, n)$, then there exists $\left(b_{m}\right)_{m \in M_{3}}, \xi$-faithful for $(\delta, L, n)$, and such that

$$
\sum_{m \in M_{4} \backslash \bigcup_{i=1}^{n} F_{i}^{\xi}(L)}\left|b_{m}\right|<\epsilon
$$

Finally, choose $M_{5} \in\left[M_{4}\right]$ according to Lemma 4.5 applied for " $N "=M_{4}$. It follows now, by (3), that for every $L \in\left[M_{5}\right]$ and $n \in \mathbb{N}$, there exists a scalar 
sequence $\left(a_{m}\right)_{m \in M_{3}}$ which is $\xi$-faithful for $(\delta, L, n)$ and such that

$$
\sum_{m \in M_{4} \backslash \bigcup_{i=1}^{n} F_{i}^{\xi}(L)}\left|a_{m}\right|<\epsilon .
$$

Now let $Q \in\left[M_{5}\right]$. Let $\left(\zeta_{n}+1\right)$ be the sequence of ordinals associated to $\xi$. Repeated applications of Proposition 2.15now yield an increasing sequence of elements of $Q,\left(l_{i}\right)$, and a sequence $\left(Q_{i}\right)$ of infinite subsets of $Q$, so that

$$
l_{1}<\operatorname{supp} \xi_{1}^{Q_{1}}<l_{2}<\operatorname{supp} \xi_{1}^{Q_{2}}<\cdots
$$

and

$$
l_{i}\left\|\xi_{1}^{Q_{i}}\right\|_{\alpha}<\epsilon_{i} \text {, for all } \alpha \in\left\{\zeta_{m}: m \leq l_{i}\right\} \cup\{0\} \text { and } i \in \mathbb{N} \text {. }
$$

We thus obtain, by stability, $P \in[Q]$ such that $\xi_{i}^{P}=\xi_{1}^{Q_{i}}$, for all $i \in \mathbb{N}$, and therefore,

$$
\begin{aligned}
& l_{1}<\operatorname{supp} \xi_{1}^{P}<l_{2}<\operatorname{supp} \xi_{2}^{P}<\cdots \\
& l_{i}\left\|\xi_{i}^{P}\right\|_{\alpha}<\epsilon_{i}, \text { for all } \alpha \in\left\{\zeta_{m}: m \leq l_{i}\right\} \cup\{0\} \text { and } i \in \mathbb{N} .
\end{aligned}
$$

Let $K$ be the $\ell_{1}^{\xi}$ spreading model constant of $\left(x_{m}\right)_{m \in M}$. By our choice of $M_{2} \in\left[M_{1}\right]$, (11) and (51) yield that

$$
\left\|\xi_{i}^{P} \cdot s\right\| \geq K-\frac{\epsilon_{i}}{l_{i}}(K+1)
$$

and hence $\left(\xi_{i}^{P} \cdot s\right)$ is semi-normalized.

We now claim that

$$
\left\|\sum_{i \in F} \xi_{i}^{P} \cdot s\right\| \leq 2 \lambda
$$

for all $F \in[\mathbb{N}]^{<\infty}$. Our claim of course implies that $\left(\xi_{i}^{P} \cdot s\right)$ is equivalent to the unit vector basis of $c_{0}$. Were our claim false, there would exist $q \in \mathbb{N}$, integers $i_{1}<\cdots<i_{q}$, and $y^{*} \in B_{X^{*}}$ such that

$$
y^{*}\left(\xi_{i_{n}}^{P} \cdot s\right) \geq 0, \text { for } n \leq q, \text { and } \sum_{n=1}^{q} y^{*}\left(\xi_{i_{n}}^{P} \cdot s\right)>\lambda .
$$

Since $P \in\left[M_{3}\right]$, (2) yields $x^{*} \in B_{X^{*}}$ such that

$$
\begin{aligned}
& x^{*}\left(\xi_{i_{n}}^{P} \cdot s\right) \geq 0, \text { for } n \leq q, \sum_{n=1}^{q} x^{*}\left(\xi_{i_{n}}^{P} \cdot s\right)>\lambda \text { and } \\
& \sum_{m \in M_{3} \backslash \bigcup_{n=1}^{q} \operatorname{supp} \xi_{i_{n}}^{P}}\left|x^{*}\left(x_{m}\right)\right|<\epsilon .
\end{aligned}
$$

We set $I=\left\{n \leq q: x^{*}\left(\xi_{i_{n}}^{P} \cdot s\right) \geq 2 \epsilon_{i_{n}}\right\}$. It follows that

$$
\sum_{n \in I} x^{*}\left(\xi_{i_{n}}^{P} \cdot s\right)>\lambda-2 \epsilon .
$$

We now fix $n \in I$ and let

$$
E_{n}=\left\{m \in \operatorname{supp} \xi_{i_{n}}^{P}: x^{*}\left(x_{m}\right) \geq \frac{1}{2} x^{*}\left(\xi_{i_{n}}^{P} \cdot s\right)\right\} .
$$


Because of (5), Lemma 4.7 yields the existence of a maximal $S_{\xi}$ subset $D_{n}$ of $\left\{l_{i_{n}}\right\} \cup E_{n}$ such that $\min D_{n}=l_{i_{n}}$. Let $m_{0}=\max D_{\max I}$ and set

$$
L=\bigcup_{n \in I} D_{n} \cup\left\{m \in Q: m>m_{0}\right\}
$$

Since $L \in\left[M_{5}\right]$, we obtain through (44), a scalar sequence $\left(a_{m}\right)_{m \in M_{3}}$ which is $\xi$ faithful for $(\delta, L,|I|)$ and such that

$$
\sum_{m \in M_{4} \backslash \bigcup_{n \in I} D_{n}}\left|a_{m}\right|<\epsilon .
$$

We recall here that for every $n \in I$,

$$
a_{m} \geq 0, \quad \text { for all } m \in D_{n}, \sum_{m \in D_{n}} a_{m} \leq 1 \quad \text { and } \quad\left\|\sum_{m \in D_{n}} a_{m} x_{m}\right\|>\delta .
$$

Of course, $\left(a_{m} x_{m}\right)_{m \in M_{3}}$ is series bounded by 1 and $\left|a_{m}\right| \leq \frac{\delta}{2}$, for all $m \in M_{3}$. Therefore,

$$
\sum_{m \in D_{n} \backslash\left\{l_{i_{n}}\right\}} a_{m}>\frac{\delta}{2} \text {, for all } n \in I .
$$

Our construction yields that

$$
\sum_{m \in M_{3} \backslash \bigcup_{n \in I} D_{n}}\left|a_{m}\right|\left|x^{*}\left(x_{m}\right)\right|<2 \epsilon
$$

Indeed,

$$
\begin{aligned}
\sum_{m \in M_{3} \backslash \bigcup_{n \in I} D_{n}}\left|a_{m}\right|\left|x^{*}\left(x_{m}\right)\right| & \leq \sum_{m \in M_{4} \backslash \bigcup_{n \in I} D_{n}}\left|a_{m}\right|+\sum_{m \in M_{3} \backslash M_{4}}\left|x^{*}\left(x_{m}\right)\right| \\
& <\epsilon+\sum_{m \in M_{3} \backslash \bigcup_{n=1}^{q} \operatorname{supp} \xi_{i_{n}}^{P}}\left|x^{*}\left(x_{m}\right)\right|, \quad \text { by (8) }, \\
& \text { and since } \left.P \in\left[M_{4}\right],<\epsilon+\epsilon=2 \epsilon, \quad \text { by (6) }\right) .
\end{aligned}
$$

We also observe that

$$
\sum_{n \in I}\left|x^{*}\left(x_{l_{i_{n}}}\right)\right|<\epsilon, \text { as } l_{i_{n}} \in M_{3} \backslash \bigcup_{n=1}^{q} \operatorname{supp} \xi_{i_{n}}^{P} \text {, for all } n \in I \text {. }
$$


Hence,

$$
\begin{aligned}
x^{*}\left(\sum_{m \leq m_{0}} a_{m} x_{m}\right) & \geq \sum_{m \in \cup_{n \in I} D_{n}} a_{m} x^{*}\left(x_{m}\right)-\sum_{m \in M_{3} \backslash \bigcup_{n \in I} D_{n}}\left|a_{m}\right|\left|x^{*}\left(x_{m}\right)\right| \\
& >\sum_{n \in I} \sum_{m \in D_{n}} a_{m} x^{*}\left(x_{m}\right)-2 \epsilon \text {, by (10), } \\
& \geq \sum_{n \in I} \sum_{m \in D_{n} \backslash\left\{l_{i_{n}}\right\}} a_{m} x^{*}\left(x_{m}\right)-\sum_{n \in I}\left|x^{*}\left(x_{l_{i_{n}}}\right)\right|-2 \epsilon \\
& \geq \sum_{n \in I} \sum_{m \in D_{n} \backslash\left\{l_{i_{n}}\right\}} a_{m} \frac{1}{2} x^{*}\left(\xi_{i_{n}}^{P} \cdot s\right)-3 \epsilon \text {, by (111), } \\
& \text { and } \operatorname{since} D_{n} \backslash\left\{l_{i_{n}}\right\} \subset E_{n}, \\
& \geq \frac{\delta}{4} \sum_{n \in I} x^{*}\left(\xi_{i_{n}}^{P} \cdot s\right)-3 \epsilon, \text { by (9) } \\
& \geq \frac{\delta}{4}(\lambda-2 \epsilon)-3 \epsilon, \text { by (7). }
\end{aligned}
$$

It now follows that

$$
1 \geq\left\|\sum_{m \leq m_{0}} a_{m} x_{m}\right\|>\frac{\delta}{4}(\lambda-2 \epsilon)-3 \epsilon,
$$

and thus $\lambda<\frac{4}{\delta}(4 \epsilon+1)$ contradicting the choice of $\lambda$.

Hence, our claim holds and $\left(\xi_{i}^{P} \cdot s\right)$ is equivalent to the unit vector basis of $c_{0}$. Moreover, the equivalence constant $C$, depends only on $K, \delta$ and $\epsilon$. It is now easily seen that the set

$$
\left\{P \in\left[M_{5}\right]:\left(\xi_{i}^{P} \cdot s\right) \text { is } C \text {-equivalent to the } c_{0} \text {-basis }\right\},
$$

is closed in $\left[M_{5}\right]$ and therefore Ramsey. Our previous argument yields $N \in\left[M_{5}\right]$ so that $\left(\xi_{i}^{Q} \cdot s\right)$ is equivalent to the unit vector basis of $c_{0}$, for all $Q \in[N]$. The proof of Theorem 1.5 is now complete.

Theorem 1.4 follows immediately from Theorem 1.5

\section{Non-trivial weak Cauchy sequences}

The last section is devoted to the relation between Theorems 1.4 and 1.6 We first observe the following immediate consequence of Theorem (1.6)

Corollary 5.1. The following are equivalent for a non-trivial weak Cauchy sequence $\left(x_{n}\right)$ in a Banach space:

1. There exists a subsequence of $\left(x_{n}\right)$ which is (s.s.).

2. There exists a subsequence $\left(x_{m_{n}}\right)$ of $\left(x_{n}\right)$ such that every convex block subsequence of $\left(x_{m_{n}}\right)$ is semi-boundedly complete.

Proof. The fact that 1 implies 2 is immediate since (s.s.) sequences are easily seen to be semi-boundedly complete, and every convex block subsequence of an (s.s) sequence is also (s.s) $[\mathrm{R}$.

Suppose now that 2 holds. If no subsequence of $\left(x_{n}\right)$ is (s.s), then Theorem (1.6) yields a convex block subsequence of $\left(x_{m_{n}}\right)$ equivalent to the summing basis. But the summing basis is not semi-boundedly complete. This contradiction shows that 1 must hold. 
Corollary 5.1 yields the following equivalent formulation of Rosenthal's theorem:

Corollary 5.2. For a non-trivial weak Cauchy sequence $\left(x_{n}\right)$ in a Banach space, one of the following statements holds exclusively:

1. There exists a subsequence $\left(x_{m_{n}}\right)$ of $\left(x_{n}\right)$ such that every convex block subsequence of $\left(x_{m_{n}}\right)$ is semi-boundedly complete.

2. Every subsequence of $\left(x_{n}\right)$ admits a convex block subsequence equivalent to the summing basis.

Evidently, Corollary 5.2 makes even more transparent the analogy between Rosenthal's result and Theorem 1.4. We also note here that, as it is shown in $\left[\mathrm{R},\left(x_{n}\right)\right.$ is an (s.s) sequence, if and only if every proper subsequence of its difference sequence is semi-boundedly complete. (In the terminology of $[\mathrm{R}]$, the difference sequence $\left(e_{i}\right)$ of an (s.s) sequence is (c.c.). That is, if $\sup _{n}\left\|\sum_{i=1}^{n} a_{i} e_{i}\right\|$ is finite, then the scalar sequence $\left(a_{n}\right)$ converges.)

We next give a quantitative version of Theorem 1.6

Corollary 5.3. Let $t=\left(x_{n}\right)$ be a non-trivial weak Cauchy sequence having no subsequence which is (s.s.). Then for every $N \in[\mathbb{N}]$ there exist $M \in[N]$, a countable ordinal $\xi$ and a constant $C>0$, so that $\left(\xi_{n}^{L} \cdot t\right)$ is $C$-equivalent to the summing basis for every $L \in[M]$.

The proof of this corollary requires the following lemma.

Lemma 5.4. Let $\xi$ be a countable ordinal and $P \in[\mathbb{N}]$. Let $t=\left(x_{n}\right)$ be a sequence in a Banach space which is $(L, \xi)$ convergent (Definition 2.2), for every $L \in[P]$. Given $\left(\epsilon_{n}\right)$, a sequence of positive scalars, there exists $M \in[P]$ such that $\left\|\xi_{n}^{L} \cdot t\right\|<$ $\epsilon_{n}$, for every $L \in[M]$ and all $n \in \mathbb{N}$.

Proof. Let $Q \in[P]$. Our assumptions allow us to choose $\left(k_{n}\right)$, an infinite subset of $\mathbb{N}$, so that $\left\|\xi_{k_{n}}^{Q} \cdot t\right\|<\epsilon_{n}$, for all $n \in \mathbb{N}$. Stability now yields $L \in[Q]$ such that $\left\|\xi_{n}^{L} \cdot t\right\|<\epsilon_{n}$, for all $n \in \mathbb{N}$. The assertion of the lemma follows from this since $\left\{L \in[P]:\left\|\xi_{n}^{L} \cdot t\right\|<\epsilon_{n}\right.$, for all $\left.n \in \mathbb{N}\right\}$ is a closed subset of $[P]$ and therefore Ramsey.

Proof of Corollary 5.3 Theorem (1.6) yields $u=\left(u_{n}\right)$ a convex block subsequence of $\left(x_{n}\right)$ equivalent to the summing basis. We set $v=\left(x_{n}-u_{n}\right)$ which is clearly a weakly null sequence. Employing the results of $[\mathrm{A}-\mathrm{A}$ ], we find a countable ordinal $\xi$ such that no subsequence of $v$ is an $\ell_{1}^{\xi}$ spreading model. It follows now, by Theorem 1.7, that there exists $P \in[N] \operatorname{such}$ that $\lim _{n}\left\|\xi_{n}^{L} \cdot v\right\|=0$, for every $L \in[P]$. We next choose $\left(\epsilon_{n}\right)$, a sequence of positive scalars such that $\sum_{n} \epsilon_{n}<1$. As a consequence of Lemma 5.4 we obtain $M \in[P]$ such that $\left\|\xi_{n}^{L} \cdot v\right\|<\epsilon_{n}$, for every $L \in[M]$ and all $n \in \mathbb{N}$. A standard perturbation result now yields $D>0$ such that $\left(\xi_{n}^{L} \cdot t\right)$ is $D$-equivalent to $\left(\xi_{n}^{L} \cdot u\right)$, for all $L \in[M]$. Since the summing basis is uniformly equivalent to all of its convex block subsequences, we obtain $C>0$ such that $\left(\xi_{n}^{L} \cdot t\right)$ is $C$-equivalent to the summing basis, for all $L \in[M]$. This completes the proof.

We observe the similarity between the statements of Theorem 1.5 and Corollary 5.3. However, the set of ordinals $\xi$ satisfying the conclusion of Theorem [1.5] is a bounded segment of $\left[0, \omega_{1}\right)$, in contrast with the corresponding set in Corollary 5.3 which is of course unbounded. 
We continue our discussion about the relation between Theorems 1.4 and 1.6 Recall that Rosenthal's $c_{0}$-theorem states that every non-trivial weak Cauchy sequence $\left(x_{j}\right)$, either has an (s.s) subsequence, or else there exists a convex block subsequence $\left(s_{j}\right)$ of $\left(x_{j}\right)$ equivalent to the summing basis. In the later case, setting $v_{j}=x_{j}-s_{j}$, we observe that $\left(v_{j}\right)$ is weakly null and that $\left(x_{j}-v_{j}\right)$ is equivalent to the summing basis. Therefore, passing to a convex block subsequence in Rosenthal's theorem acts as a filtration to remove the "noise" coming from an arbitrary weakly null sequence. In our case the reasoning for passing to a convex block subsequence is different: It exhausts the local $\ell_{1}$ structure of the sequence.

In spite of these differences it seems that there are similarities in the statements for weakly null and non-trivial weak Cauchy sequences. Our final corollary which is the analog to Elton's dichotomy illustrates this.

Corollary 5.5. For a non-trivial weak Cauchy sequence $\left(x_{n}\right)$ one of the following statements holds exclusively:

1. Every subsequence of $\left(x_{n}\right)$ admits a subsequence equivalent to the summing basis.

2. There exists a subsequence of $\left(x_{n}\right)$ which is semi-boundedly complete.

Proof. Clearly the statements are mutually exclusive since the summing basis is not semi-boundedly complete.

Suppose that 2. does not hold. It follows that no subsequence of $\left(x_{n}\right)$ is (s.s). Theorem 1.6 now yields that every subsequence of $\left(x_{n}\right)$ admits a convex block subsequence equivalent to the summing basis. To prove that 1 holds let $\left(x_{m_{n}}\right)$ be a subsequence of $\left(x_{n}\right)$. Without loss of generality, by passing to a subsequence according to Proposition 2.2 of $[\mathbb{R}]$, we can assume that $\left(x_{m_{n}}\right)$ dominates the summing basis.

Next choose $\left(u_{n}\right)$, a convex block subsequence of $\left(x_{n}\right)$ equivalent to the summing basis. We set $y_{n}=x_{m_{n}}-u_{n}$, for all $n \in \mathbb{N}$. If $\left(y_{n}\right)$ is not semi-normalized, then 1 follows. So assuming that $\left(y_{n}\right)$ is semi-normalized we claim that there exists a subsequence of $\left(y_{n}\right)$ equivalent to the unit vector basis of $c_{0}$. Indeed, if that were not the case, then by Elton's dichotomy, Corollary [3.3. there would exist a semiboundedly complete subsequence of $\left(y_{n}\right)$. But since every subsequence of $\left(x_{m_{n}}\right)$ dominates the summing basis (and therefore every subsequence of $\left(u_{n}\right)$ as well), we obtain that $\left(x_{m_{n}}\right)$ has a semi-boundedly complete subsequence which of course contradicts our assumption that 2 does not hold. Hence, our claim must hold and it immediately yields a subsequence of $\left(x_{m_{n}}\right)$ equivalent to the summing basis in view of the following elementary fact: Let $\left(f_{n}\right)$ and $\left(g_{n}\right)$ be sequences in a Banach space with $\left(f_{n}\right)$ equivalent to the summing basis and $\left(g_{n}\right)$ equivalent to the $c_{0}$ basis. Then there exists a subsequence of $\left(f_{n}+g_{n}\right)$ equivalent to the summing basis.

\section{REFERENCES}

[A-A] D. Alspach and S.A. Argyros, Complexity of weakly null sequences, Dissertationes Mathematicae 321 (1992), 1-44. MR 93j:46014

[A-O] D. Alspach and E. Odell, Averaging weakly null sequences, Lecture Notes in Math. 1332 Springer (1988), 126-143. MR 89j:46014

[AN-O] G. Androulakis and E. Odell, Distorting mixed Tsirelson spaces, Israel J. Math. 109 (1999), 125-149. MR 2000f: 46012

[A-M-T] S.A. Argyros, S. Mercourakis and A. Tsarpalias, Convex unconditionality and summability of weakly null sequences, Israel J. Math. 107 (1998), 157-193. MR 99m:46021 
[A-F] S.A. Argyros and V. Felouzis, Interpolating hereditarily indecomposable Banach spaces, J. Amer. Math. Soc. 13 (2000), 243-294. CMP 2000:11

[B-P] C. Bessaga and A. Pelczynski, On bases and unconditional convergence of series in Banach spaces, Studia Math., 17 (1958) 151-164. MR 22:5872

[C] P. Cembranos, The hereditary Dunford-Pettis property on $C(K, E)$, Illinois J. Math. 31 (1987), 365-373. MR 88g:46028

[E] J. Elton, Thesis, Yale Univ. (1978).

[Ell] E. Ellentuck, A new proof that analytic sets are Ramsey, J. Symbolic Logic 39 (1974), 163-165. MR 50:1887

[G-P] F. Galvin and K. Prikry, Borel sets and Ramsey's theorem, J. Symbolic Logic 38 (1973), 193-198. MR 49:2399

[K-O] H. Knaust and E. Odell, On $c_{0}$ sequences in Banach spaces, Israel J. Math. 67 (1989), 153-169. MR 91d:46013

[M-R] B. Maurey and H.P. Rosenthal, Normalized weakly null sequence with no unconditional subsequence, Studia Math. 61 (1977), 77-98. MR 55:11010

[NW] C. St. J. A. Nash-Williams, On well-quasi-ordering transfinite sequences, Proc. Camb. Phil. Soc. 61 (1965), 33-39. MR 30:3850

[O1] E. Odell, Applications of Ramsey theorems to Banach space theory, Notes in Banach spaces, (H.E. Lacey ed.), Univ. of Texas Press (1980), 379-404. MR 83g:46018

[O2] E. Odell, On Schreier unconditional sequences, Contemporary Math. 144 (1993), 197201. MR 94f:56016

[O-S] E. Odell and Th. Schlumprecht, A problem on spreading models, J. Funct. Anal. 153 (1998), 249-261. MR 99c:46006

[R] H.P. Rosenthal, A characterization of Banach spaces containing $c_{0}$, J. Amer. Math. Soc. 7 (1994), 707-748. MR 94i:46032

[Sc] J. Schreier, Ein Gegenbeispiel zur theorie der schwachen konvergenz, Studia Math. 2 (1930), 58-62.

[Si] J. Silver, Every analytic set is Ramsey, J. Symbolic Logic 35 (1970), 60-64. MR 48:10807

[Sz] W. Szlenk, The non-existence of a separable reflexive Banach space universal for all separable reflexive Banach spaces, Studia Math. 30 (1968), 53-61. MR 37:3327

[T] A. Tsarpalias, A note on Ramsey property, Proc. A.M.S. 127 (1999), no. 2, 583-587. MR 99c:04005

Department of Mathematics, University of Athens, Athens 15784, Greece

Current address: Department of Mathematics, National Technical University of Athens, 15780 Athens, Greece

E-mail address: sargyros@math.ntua.gr

Department of Mathematics, Oklahoma State University, Stillwater, Oklahoma 74078-1058

E-mail address: ioagaspa@math.okstate.edu 